\title{
The Role of Feedback in Shaping the Extra-Classical Receptive Field of Cortical Neurons: A Recurrent Network Model
}

\author{
Lars Schwabe, ${ }^{1,2,3}$ Klaus Obermayer, ${ }^{1}$ Alessandra Angelucci, ${ }^{2 *}$ and Paul C. Bressloff ${ }^{3 *}$ \\ ${ }^{1}$ Fakultät IV, Electrical Engineering and Computer Science, Technische Universität Berlin, 10587 Berlin, Germany, ${ }^{2}$ Department of Ophthalmology and \\ Visual Science, Moran Eye Center, University of Utah, Salt Lake City, Utah 84132, and '3Department of Mathematics, University of Utah, Salt Lake City, Utah \\ 84112
}

The responses of neurons in sensory cortices are affected by the spatial context within which stimuli are embedded. In the primary visual cortex (V1), orientation-selective responses to stimuli in the receptive field (RF) center are suppressed by similarly oriented stimuli in the RF surround. Surround suppression, a likely neural correlate of perceptual figure-ground segregation, is traditionally thought to be generated within V1 by long-range horizontal connections. Recently however, it has been shown that these connections are too short and too slow to mediate fast suppression from distant regions of the RF surround. We use an anatomically and physiologically constrained recurrent network model of macaque $\mathrm{V} 1$ to show how interareal feedback connections, which are faster and longer-range than horizontal connections, can generate "far" surround suppression. We provide a novel solution to the puzzle of how surround suppression can arise from excitatory feedback axons contacting predominantly excitatory neurons in V1. The basic mechanism involves divergent feedback connections from the far surround targeting pyramidal neurons sending monosynaptic horizontal connections to excitatory and inhibitory neurons in the RF center. One of several predictions of our model is that the "suppressive far surround" is not always suppressive, but can facilitate the response of the RF center, depending on the amount of excitatory drive to the local inhibitors. Our model provides a general mechanism of how top-down feedback signals directly contribute to generating cortical neuron responses to simple sensory stimuli.

Key words: primary visual cortex; macaque; horizontal connections; top-down; contextual effects; surround suppression

\section{Introduction}

Neuronal responses in sensory cortices are modulated by the spatial context within which stimuli are embedded. Primary visual cortex (V1) neuron responses to optimally oriented stimuli in their receptive field (RF) center are usually suppressed by isooriented stimuli in the RF surround (Blakemore and Tobin, 1972; Nelson and Frost, 1978; Allman et al., 1985; Gilbert and Wiesel, 1990; DeAngelis et al., 1994). V1 cells also exhibit contrastdependent size-tuning (Sengpiel et al., 1997; Kapadia et al., 1999; Sceniak et al., 1999) (i.e., the radius of a stimulus evoking the largest response from the cell is smaller at high than at low contrast). We refer to the optimal radius for spatial summation as the high- or low-contrast summation RF (hsRF or lsRF, respectively). The RF center and surround have a number of distinct components, as defined in Figure 1. In particular, we distinguish

\footnotetext{
Received March 23, 2006; revised June 9, 2006; accepted July 21, 2006.

This work was supported by the Wellcome Trust (061113), National Institutes of Health (EY 015262), National Science Foundation (IBN 0344569 and DMS 0515725), Research to Prevent Blindness, and BMBF (10025304). We thank Dr. Jennifer $S$. Lund for her intellectual contribution to this study.

${ }^{*}$ A.A. and P.C.B. contributed equally to this work.

Correspondence should be addressed to Alessandra Angelucci at the above address. E-mail: alessandra.angelucci@hsc.utah.edu.

DOI:10.1523/JNEUROSCI.1253-06.2006

Copyright $\odot 2006$ Society for Neuroscience $\quad$ 0270-6474/06/269117-13\$15.00/0
}

between a "near" surround that extends as far as the lsRF, and a suppressive "far" surround extending beyond the lsRF.

Previous studies (Angelucci et al., 2002b) demonstrated that V1 horizontal connections are spatially coextensive with the lsRF size of V1 cells (Fig. 1), and that surround suppression can be evoked by stimuli located far beyond the monosynaptic spread of horizontal connections (Sceniak et al., 2001; Cavanaugh et al., 2002a; Levitt and Lund, 2002). Thus, these connections could monosynaptically underlie the spatial summation properties of V1 neurons at low stimulus contrast (Sceniak et al., 1999) and near (Kapadia et al., 1995; Polat et al., 1998), but not far, surround modulation of RF center responses. Cascades of horizontal connections are too slow (Grinvald et al., 1994; Bringuier et al., 1999; Girard et al., 2001) to account for the fast onset of suppression from the far surround (Bair et al., 2003; Muller et al., 2003). The latter could instead be accounted for by longer-range (Angelucci et al., 2002b) and faster-conducting (Girard et al., 2001) extrastriate feedback (FB) connections to V1. Consistent with the hypothesis that FB connections could underlie the far surround of V1 neurons, is evidence that cooling the middle temporal (MT) area reduces the suppressive effect of surround motion stimulation in V3, V2, and V1 neurons (Hupé et al., 1998; Bullier et al., 2001). However, a role for FB connections in surround suppression is inconsistent with evidence that these connections 
arise from excitatory neurons, target almost exclusively (97-98\%) excitatory V1 neurons (Johnson and Burkhalter, 1996), and modulate V1 responses via synaptic mechanisms in which excitation dominates (Shao and Burkhalter, 1996).

To pinpoint the neuronal circuitry and mechanisms underlying center-surround interactions in macaque V1, we have set up a recurrent neuronal network model whose architecture is constrained to fit the anatomical and physiological data described above. In particular, we provide a novel solution to how excitatory $\mathrm{FB}$ connections could mediate far surround suppression in V1, namely by targeting excitatory neurons sending monosynaptic horizontal connections to local inhibitors in the RF center. This is consistent with observations that horizontal connections, unlike FB connections, terminate on both excitatory and inhibitory neurons (McGuire et al., 1991). Our model can account for a wide range of physiological data regarding the static and dynamic effects of surround suppression, and generates novel testable predictions. One important prediction, consistent with our recent studies (Ichida et al., 2005), is that the far suppressive surround can be facilitatory.

\section{Materials and Methods}

We describe here the construction of our cortical network model and how the parameters of the model are fitted to anatomical and physiological data. The values of all model parameters are summarized in Table 1.

Cortical network model. We consider a recurrent network model that represents two areas of an idealized cortex, one corresponding to area V1 (or striate cortex) and the other to an extrastriate area such as MT, with each area being composed of a single layer of cells, namely layer $2 / 3$ of V1 and layer 6 of MT. A schematic diagram illustrating the basic network architecture is shown in Figure 2. Suppose that a particular V1 excitatory neuron $\left(E_{\mathrm{ctr}}\right)$ is identified as the target neuron whose response properties we wish to determine. V1 neurons can then be labeled according to the location (in degrees) of their RF centers relative to the RF center of the target neuron. Thus, the "center" consists of neurons $\left(E_{\mathrm{ctr}}\right)$ whose RF centers lie within the minimum response field ( $\mathrm{mRF}$ ) of the target neuron, the "near surround" consists of neurons $\left(E_{\mathrm{nr}}\right)$ whose RF centers fall within either the hsRF or lsRF, and the "far surround" consists of neurons $\left(E_{\text {far }}\right)$ whose RF centers lie beyond the lsRF (Fig. 1). In the model, we assume that excitatory neurons in $\mathrm{V} 1(E)$ receive feedforward afferent excitation $\left(E_{\mathrm{FF}}\right)$ from other V1 layers, local recurrent excitation and inhibition ( $I$ ), long-range excitation via slow intra-areal V1 horizontal (or lateral) connections, and $\mathrm{FB}$ excitation $\left(E_{\mathrm{FB}}\right)$ via fast interareal connections. Horizontal connections also target local interneurons, whereas FB connections only target excitatory neurons. This is motivated by the anatomical findings that horizontal axons contact both excitatory $(80 \%)$ and inhibitory (20\%) neurons (McGuire et al., 1991), whereas FB axons contact predominantly (97-98\%) excitatory neurons (Johnson and Burkhalter, 1996) (see Introduction). The spatial extent of interareal FB connections is taken to be larger than that of intra-areal horizontal connections (Fig. 3a), following the anatomical data of Angelucci et al. (2002b). In particular, horizontal connections from the near surround, but not the far surround, connect to the RF center monosynaptically, and extend as far as the lsRF of their neurons of origin, whereas extrastriate FB connections are coextensive with the far surround of V1 neurons.

The fundamental assumption of our model is that there is an asymmetry between the properties of the excitatory cells and those of the local interneurons, namely, that the local inhibitory neurons have higher firing thresholds and gain (Fig. $3 b$ ), and are more strongly driven by horizontal connections than the excitatory neurons whose output they control. As a result, the inhibitory neurons generate suppression under sufficiently high levels of excitation, but are inactive at low levels of excitation. This is consistent with the results of electrical stimulation experiments in slices of visual cortex showing that weaker stimulation of horizontal connections evokes excitation in their target cells, whereas inhibition dominates after stronger stimulation (Hirsch and Gilbert, 1991). There is experimental evidence for high-gain inhibitory neurons in cortical layer $2 / 3$. These are interneurons receiving facilitating inputs from pyramidal cells (Thomson et al., 1995, 2002; Markram et al., 1998; Thomson, 2003), unlike other interneurons also targeted by pyramidal cells, which instead receive depressing inputs. Whereas depressing interneurons receive inputs only from the local pyramids that they contact, facilitating interneurons receive inputs also from other pyramids and, thus, perhaps from those sending horizontal connections (Pouille and Scanziani, 2004; Kapfer et al., 2005) (M. Scanziani, personal communication). Although the existence of higher-threshold inhibitory neurons strongly driven by horizontal connections has not been confirmed experimentally, there exist a large number of different classes of interneurons, many of whose physiological properties have not yet been fully characterized. Similarly, we know very little about the properties of inhibitory neurons targeted by horizontal connections. Therefore, this basic assumption of our model can be viewed as an experimentally verifiable hypothesis that provides a simple explanation for a wide range of phenomena, as described in detail in Results. It should also be noted that the inhibitory neurons with facilitating synapses have been shown to be recruited in the late phase of the action potential train of their presynaptic pyramidal cells (Pouille and Scanziani, 2004; Kapfer et al., 2005). The latter could thus represent a mechanism alternative to the higherthreshold interneurons we proposed to implement the delayed recruitment of inhibition relative to excitation needed in our model.

For simplicity, we do not model stimulus features such as orientation and spatial frequency, because in this paper we focus on modeling the size tuning of V1 neurons and how this is affected by stimulus contrast. Thus, we model experimental paradigms in which two-dimensional gratings are presented at the optimal spatial frequency and orientation of the center target neuron. In real life, such stimuli activate discrete patches across the cortex corresponding to those cortical columns that have sufficiently similar orientations and spatial frequencies to that of the stimulus. We idealize this patchwork structure as a one-dimensional network of cells along the collinear axis of the preferred orientation, in agreement with recent data showing that horizontal (Bosking et al., 1997; Sincich and Blasdel, 2001) and FB (Angelucci et al., 2003; Shmuel et al., 2005) connections in V1 link regions of similar orientation preference along an axis collinear with the preferred orientation of their neurons of origin.

As a further simplification, we consider a spatially discrete model in which each cortical area is represented as a one-dimensional lattice of excitatory/inhibitory (E-I) neuron pairs whose RF centers are equally spaced between -8 and $+8^{\circ}$. Let $N^{\alpha}$ be the total number of E-I pairs in the striate $(\alpha=S)$ and extrastriate $(\alpha=X)$ cortex. The RF centers (in degrees) can then be written as $x_{i}=8 i / M^{\alpha}$, with $i=0, \pm 1, \ldots, \pm M^{\alpha}$, and $N^{\alpha}=2 M^{\alpha}+1$. In our simulations we take $N^{S}=161$ and $N^{X}=33$. It follows that the RF centers of neighboring E-I pairs in the striate cortex are spatially separated by $0.1^{\circ}$, which is equivalent to $230 \mu \mathrm{m}$ in cortical distance at $5^{\circ}$ retinal eccentricity (see below, Spatial scales and time delays), and is consistent with the approximate interpatch distance of horizontal intracortical connections. Given this spatially coarse-grained representation of the cortex, the center is represented by the target E-I pair together with its nearest and next-nearest neighbors, assuming the mRF has a radius of $\sim 0.2^{\circ}$. One can think of each E-I pair as the basic functional module of our network that incorporates the effects of local recurrent connections. Interactions between these modules are then mediated by horizontal and feedback connections. Because we are focusing on size-tuning effects in this paper, it is sufficient to take a very simple local network model. However, if one were to extend the model to incorporate orientation tuning, for example, then it would be necessary to replace each E-I pair with a more complicated network module such as one based on the ring model (Ben-Yishai et al., 1995; Somers et al., 1995). In this case, at least two distinct types of inhibitory neuron would be needed (Stetter et al., 2000; Bressloff and Cowan, 2002), one that is broadly tuned with respect to orientation and one that is more sharply tuned, with the latter playing the role of the local inhibitor in our simpler model. Anatomically speaking, these two classes of interneurons could be identified, respectively, as laterally projecting basket cells and vertically projecting cells, for example, double bouquet cells.

Network dynamics. The cortical network dynamics is formulated in terms of a simplified rate model, which can be derived from a more 
detailed biophysical model as described previously (Shriki et al., 2003). Let $r_{l}{ }^{\alpha}\left(x_{i}, t\right)$ denote the local activity at time $t$ of excitatory $(l=E)$ and inhibitory $(l=I)$ neurons with their RF center at $x_{i}$ in the striate $(\alpha=S)$ and extrastriate $(\alpha=X)$ cortex. The activity $r_{l}{ }^{\alpha}$ evolves according to the first-order rate equation as follows:

$$
\tau_{l} \frac{d r_{l}^{\alpha}\left(x_{i}, t\right)}{d t}=-r_{l}^{\alpha}\left(x_{i}, t\right)+F_{l}^{\alpha}\left(I_{l}^{\alpha}\left(x_{i}, t\right)\right)
$$

where $\tau_{l}$ is an effective synaptic time constant (with $\tau_{l}=8 \mathrm{~ms}$ ), $F_{l}{ }^{\alpha}(I)$ is the firing rate function or frequency-current curve for the cell (see below, Firing rate function), and $I_{l}^{\alpha}\left(x_{i}, t\right)$ is the total synaptic current into the neuron. In the striate cortex, the synaptic current is taken to be the following:

$$
\begin{aligned}
I_{l}^{S}\left(x_{i}, t\right) & =\frac{\sum_{m=E, I} W_{l m}^{S} r_{m}^{S}\left(x_{i}, t\right)}{\text { local inputs }}+\frac{\sum_{j \neq i} W_{l E}^{S S}\left(x_{i} \mid x_{j}\right) \cdot r_{E}^{S}\left(x_{j}, t-\Delta t^{S S}\left(x_{i} \mid x_{j}\right)\right)}{\text { lateral inputs within striate cortex }} \\
& +\frac{\left.\sum_{j} W_{I E}^{S X}\left(x_{i} \mid x_{j}\right) \cdot r_{E}^{X}\left(x_{j}, t-\Delta t^{S X}\left(x_{i} \mid x_{j}\right)\right)+x_{i}, t\right) \delta_{l, E}}{\text { feedback inputs from extrastriate cortex }}, \underbrace{h}_{\text {afferent inputs }},
\end{aligned}
$$

whereas in extrastriate cortex it is of the following form:

$$
\begin{aligned}
I_{i}^{X}\left(x_{i}, t\right)= & \frac{\sum_{m=E, I} W_{l m}^{X} r_{m}^{X}\left(x_{i}, t\right)}{\text { local inputs }}+\frac{\sum_{j \neq i} W_{I E}^{X X}\left(x_{i} \mid x_{j}\right) \cdot r_{E}^{X}\left(x_{j}, t-\Delta t^{X X}\left(x_{i} \mid x_{j}\right)\right)}{\text { lateral inputs within striate cortex }} \\
+ & \frac{\sum_{j} W_{I E}^{X S}\left(x_{i} \mid x_{j}\right) \cdot r_{E}^{S}\left(x_{j}, t-\Delta t^{X S}\left(x_{i} \mid x_{j}\right)\right)}{\text { feedforward inputs from striate cortex }}
\end{aligned}
$$

Here $h\left(x_{i}, t\right)$ represents the afferent input to the excitatory cell with RF center $x_{i}$ in striate cortex, $W_{l m}{ }^{\alpha}$ denotes the weight of the local synaptic connection from neuron of type $m$ to neuron of type $l$ within the same E-I pair of a given cortical area $\alpha, W_{l m}{ }^{\alpha \beta}\left(x_{i} \mid x_{j}\right)$ denotes the non-local connections from an E-I pair with RF center $x_{j}$ in area $\beta$ to an E-I pair with RF center $x_{i}$ in area $\alpha$, and $\Delta t^{\alpha \beta}\left(x_{i} \mid x_{j}\right)$ represents the corresponding axonal time delays. Thus, $W_{l m}{ }^{S S}$ and $W_{l m}{ }^{X X}$ represent the spatial distribution of intra-areal (horizontal) connections, whereas $W_{l m}^{X S}$ and $W_{l m}{ }^{S X}$ represent the distribution of interareal feedforward and feedback connections, respectively. The non-local weights depend on the distance between the RF centers $x_{i}$ and $x_{j}$ of the presynaptic and postsynaptic neurons and are given by the following (Fig. $3 a$ ):

$$
W_{l E}^{\alpha \beta}\left(x_{i} \mid x_{j}\right)=\bar{W}_{l E}^{\alpha \beta} \cdot \exp \left(-\lambda^{\alpha \beta}\left|x_{i}-x_{j}\right|\right),
$$

where $\lambda^{\alpha \beta}$ determines the spatial scale of the connections. The values of the weight coefficients $W_{l m}{ }^{\alpha}, \bar{W}_{l E}^{\alpha \beta}$, and space constants $\lambda^{\alpha \beta}$ are listed in Table 1. For simplicity, all recurrent connections in the extrastriate cortex are set to zero $\left(\bar{W}_{l E}^{X X}=0=W_{l m}^{X}\right)$ so that the latter effectively serves as a relay station without further processing.

The conduction time delays are given by the following:

$$
\Delta t^{\alpha \beta}\left(x_{i} \mid x_{j}\right)= \begin{cases}\frac{\left|x_{i}-x_{j}\right|}{\nu_{S \rightarrow S}} & \text { for lateral connections in striate cortex } \\ \frac{\Delta x_{S \rightarrow X}}{\nu_{S \rightarrow X}} & \text { for inter-areal connections, }\end{cases}
$$

where $v_{S \rightarrow S}$ and $v_{S \rightarrow X}$ are the conduction velocities for signaling within the striate cortex and between the striate and extrastriate areas, respectively, and $\Delta x_{S \rightarrow X}$ is the distance between the two areas. The values assigned to $\lambda^{\alpha \beta}$ and the conduction velocities are discussed below in Spatial scales and time delays.

Afferent inputs. The model neurons in the recurrent network correspond to neurons in layer $2 / 3$ of area V1. Therefore, the afferent input to these neurons arises from layer 4C. Let $c(y)$ denote the contrast of the stimulus at the visual field location $y$. A typical contrast-dependent output current from a layer $4 \mathrm{C}$ neuron can be approximated by a piecewise linear function of the following form:

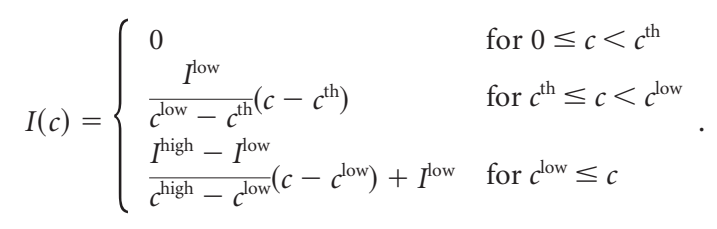

Below the contrast threshold $c^{\text {th }}$ the current is zero, whereas above threshold the current increases linearly with contrast at a rate that changes when $c$ crosses $c^{\text {low }}$. A graph of this function is shown in Figure $3 c$. The parameter values for the contrast-dependence of the input currents are set to $c^{\text {th }}=0.1, c^{\text {low }}=0.15, c^{\text {high }}=0.85, I^{\text {low }}=0.58 \mathrm{nA}$, and $I^{\text {high }}=0.71 \mathrm{nA}$. The total input to the $i^{\text {th }}$ excitatory neuron with the RF center at $x_{i}$ is then given as a linear spatial summation over the distribution of afferent inputs from layer $4 \mathrm{C}$ :

$$
h\left(x_{i}\right)=\int G\left(y ; x_{i}\right) \cdot I(c(y)) d y .
$$

Here $G\left(y ; x_{i}\right)$ represents the RF profile of the layer $2 / 3$ neuron and is taken to be a Gaussian:

$$
G\left(y ; x_{i}\right)=\left(\sqrt{2 \pi} \sigma_{a f f}\right)^{-1} \exp \left(-\left(y-x_{i}\right)^{2} /\left(2 \sigma_{a f f}^{2}\right)\right),
$$

with range $\sigma_{\text {aff }}=0.1^{\circ}$ (Bauer et al., 1999; Angelucci et al., 2002a). It follows, from the form of $I(c)$, that a necessary condition for a layer $2 / 3$ neuron to fire is that the contrast of the afferent stimulus is higher than $c^{\text {th }}$.

Firing rate function. To obtain a biophysically reasonable form for the firing rate functions $F_{E}$ and $F_{I}$, we used a Hodgkin-Huxley-type neuron with fluctuating background inputs represented by an Ornstein-Uhlenbeck process, as described in detail previously (Destexhe et al., 2001; Schwabe and Obermayer, 2005). We simulated the spike response of this model neuron to current injections for different values of the leak conductance in the presence of the fluctuating background conductance. We considered only the already adapted responses, which were then best fitted with thresholded polynomials. These fits were performed by minimizing the mean squared error between the simulated firing rate and the one predicted by the thresholded polynomials. We obtained the following:

$$
\begin{gathered}
F_{E}(I)=\max \left(0, a_{E}\left(I-\Delta I_{E}\right)\right) \\
F_{I}(I)=\max \left(0, a_{I}\left(I-\Delta I_{I}\right)+b_{I}\left(I-\Delta I_{I}\right)^{2}\right),
\end{gathered}
$$

with $a_{E}=70.09$ spikes/s $(\mathrm{sp} / \mathrm{s})(\mathrm{nA})^{-1}, \Delta I_{E}=0.52 \mathrm{nA}$ for excitatory neurons, and $a_{I}=131 \mathrm{sp} / \mathrm{s}(\mathrm{nA})^{-1}, b_{I}=-28 \mathrm{sp} / \mathrm{s}(\mathrm{nA})^{-2}$, and $\Delta I_{I}=0.7$ $\mathrm{nA}$ for inhibitory neurons (see also Table 1). The functions $F_{E}$ and $F_{I}$ are plotted in Figure $3 b . F_{E}$ is a linear function, because in the corresponding Hodgkin-Huxley model we included an adaptation current that linearizes the steady-state firing rate function. In contrast, the adaptation conductance in the Hodgkin-Huxley model of the inhibitory neuron was reduced to only $10 \%$ of the value used for the excitatory neuron. As a consequence, the steady-state firing rate function remained nonlinear, which we captured by using a quadratic function for the fit. The current threshold of the inhibitory neurons is taken to be higher than the one for the excitatory neurons. This can be implemented by assuming a higher leak conductance for inhibitory cells at rest or by assuming a different balance between the background excitation and inhibition for the inhibitory neuron. Having determined the steady-state firing rate, we can incorporate the effects of synaptic dynamics into a first-order rate equation.

Spatial scales and time delays. The parameters $\lambda^{\alpha \beta}$ determine the spatial scale of the connections between the areas $a$ and $b$. We used $\lambda^{S S}=2.3$ $\mathrm{deg}^{-1}$ and $\lambda^{X S}=\lambda^{S X}=0.3 \mathrm{deg}^{-1}$. These values are computed as follows. Defining the scale of the connections between the areas $a$ and $b$ as the value $\Delta x$ at which $\exp \left(-\lambda^{\alpha \beta} \Delta x\right)=0.05$, we have $\lambda^{\alpha \beta}=-(\Delta x)^{-1} \ln$ (0.05). For horizontal connections, we use $\Delta x=0.5 \times D_{(\mathrm{mm})} \times M F^{-1}=$ 
$1.3 \mathrm{deg}$, and obtain $\lambda^{S S}=2.3 \mathrm{deg}^{-1}$, where $D_{(\mathrm{mm})}=6 \mathrm{~mm}$ is the average length of horizontal connections in macaque V1 (Angelucci et al., 2002b), and $M F=2.3 \mathrm{~mm} / \mathrm{deg}$ is the cortical magnification factor (MF) in macaque $\mathrm{V} 1$ at $5^{\circ}$ eccentricity along the isopolar lines of visual field representation (Van Essen et al., 1984). In a previous study (Angelucci et al., 2002b), we found that the average visuotopic extent of horizontal connections closely matches the average extent of the lsRF of their V1 neurons of origin. For example, at $5^{\circ}$ eccentricity, the physiologically determined average size of the lsRF is $\sim 2.6^{\circ}$ in diameter, which is compatible with $2 \Delta x=2.6^{\circ}$. For the interareal connections, we use $\Delta x=0.5 \times D_{(\mathrm{mm})} \times M F^{-1}=9.89^{\circ}$, and obtain $\lambda^{X S}=\lambda^{S X}=0.3 \mathrm{deg}^{-1}$, where $D_{(\mathrm{mm})}=$ $8.9 \mathrm{~mm}$ is the average cortical extent of the neuronal fields of $\mathrm{FB}$ connections to $\mathrm{V} 1$ within area MT in the macaque (Angelucci et al., 2002b), and $M F=0.45 \mathrm{~mm} / \mathrm{deg}$ is the cortical magnification factor in macaque $\mathrm{MT}$ at $5^{\circ}$ eccentricity (Albright and Desimone, 1987). For simplification, we assume horizontal and FB connections to be translation invariant in visual space (i.e., we do not take into account variations in the extent of the connections caused by the dependence of the cortical MF on retinal eccentricity). This is done so as to avoid distortions of the predicted activation profiles along the cortical axis. In a model with an MF varying with eccentricity, surround suppression would become asymmetric, with stronger suppression from cortex representing visual field regions closer to the fovea. This is because near the fovea the MF is larger and, thus, a larger number of near surround neurons would exert their influence on the center neurons. In contrast, the converse is true for cortical regions away from the fovea. Thus, a more detailed model would predict asymmetries of surround suppression caused by these effects. However, these are likely to become relevant only for very large stimuli.

For the conduction velocities used to compute the time delays $\Delta t^{\alpha \beta}$, we use $v_{S \rightarrow X}=4000 \mathrm{~mm} / \mathrm{s}$ for the conduction velocity of interareal connections between V1 and MT in the macaque (Movshon and Newsome, 1996; Nowak and Bullier, 1997), and $\Delta x_{S \rightarrow X}=7 \mathrm{~mm}$ for the distance between macaque V1 and MT (Kennedy and Bullier, 1985; Hupé et al., 1998). For the intra-areal horizontal connections in the striate cortex, we use $v_{S \rightarrow X}=(200 \mathrm{~mm} / \mathrm{s}) \times M F^{-1}=86.9 \%$ (Grinvald et al., 1994; Bringuier et al., 1999; Girard et al., 2001), where $M F=2.3$ $\mathrm{mm} / \mathrm{deg}$ is the $\mathrm{V} 1$ cortical magnification factor at $5^{\circ}$ eccentricity. These values lead, for example, to time delays of $\Delta t=5^{\circ} /\left(86.9^{\circ} / \mathrm{s}\right)=57.5 \mathrm{~ms}$ for signals traveling a distance of $5^{\circ}$ within the model V1 compared with a delay of $\Delta t=(2 \times 7 \mathrm{~mm}) /(4000 \mathrm{~mm} / \mathrm{s})=3.5 \mathrm{~ms}$ for signals traveling a comparable distance via feedforward connections to extrastriate cortex and back to V1.

Parameterization of the model. In the model, there are three sets of parameters: the biophysical single cell parameters, the parameters for the spatial scales and time delays of the intra-areal and interareal connections, and the parameters determining the strengths of the connections (see Table 1). Whereas the first two sets of parameters are largely constrained by experimental data, little is known about the last parameter set. In a model exploration, we adjusted the connection weights such that the model accounted for all of the experimental data that we simulated in this paper. The only constraints we imposed were (1) excluding feedback to inhibitory neurons (Johnson and Burkhalter, 1996), and (2) assuming that horizontal connections to excitatory neurons are only modulatory (Hirsch and Gilbert, 1991) (i.e., feedforward inputs to excitatory neurons are a necessary condition for the neurons to generate action potentials). Hence, the feedforward and the local recurrent excitation of excitatory neurons are assumed to be strong compared with that of horizontal

Description Value

\section{Network architecture}

No. of excitatory and inhibitory neurons in striate cortex

No. of excitatory neurons in extrastriate cortex

Size of simulated visual field representation

Connectivity

Scale of lateral connections

Scale of interareal connections

Conduction velocity of lateral connections

Conduction velocity of interareal connections

Distance between V1 and extrastriate area MT

Spatial spread of afferent input

Firing rate functions

Parameter for excitatory neurons

Threshold for the excitatory neurons

Parameter for inhibitory neurons

Parameter for inhibitory neurons

Threshold for the inhibitory neurons

Strengths

Lateral excitation of excitatory neurons

Lateral excitation of inhibitory neurons

Interareal excitation

Local excitation of excitatory neurons

Local excitation of inhibitory neurons

Local inhibition of excitatory neurons

Local inhibition of inhibitory neurons
161

33

$16 \mathrm{deg}$

$2.3 \mathrm{deg}^{-1}$

$0.3 \mathrm{deg}^{-1}$

$200 \mathrm{~mm} / \mathrm{s}$

$4000 \mathrm{~mm} / \mathrm{s}$

$7 \mathrm{~mm}$

$0.1 \mathrm{deg}$

$70.09 \mathrm{sp} / \mathrm{s}(\mathrm{nA})^{-1}$

$0.52 \mathrm{nA}$

$131 \mathrm{sp} / \mathrm{s}(\mathrm{nA})^{-1}$

$-28 \mathrm{sp} / \mathrm{s}(\mathrm{nA})^{-2}$

$0.70 \mathrm{nA}$

$3.38 \times 10^{-4}$

$34 \times 10^{-4}$

$4.52 \times 10^{-4}$

$85 \times 10^{-4}$

$34 \times 10^{-4}$

$-12 \times 10^{-4}$
$-122 \times 10^{-4}$

connections. Under these constraints, we find that only assuming strong horizontal excitation of inhibitory neurons (Hirsch and Gilbert, 1991) can the model reproduce the experimental data (see above, Cortical network model, and Discussion). We also find that the strength of the feedforward input to inhibitory neurons has a minor effect on the model predictions, as long as the inhibitory neurons have a higher functional threshold than the excitatory neurons, because it is only in this regime that the model accurately predicts the contrast-dependence of surround suppression (Fig. 4a). It is for simplicity that we did not assume any feedforward input to inhibitory neurons. It should also be noted that we are only considering one class of inhibitory neuron in our simplified model. As we have already commented above, extending our model to take into account orientation tuning would require the introduction of at least one additional class of interneuron representing, for example, broadly tuned basket cells. The inclusion of a direct afferent drive from layer $4 \mathrm{C}$ to interneurons would then play a more significant role.

\section{Results}

To elucidate the role of horizontal and FB connections in generating the RF center and surround of V1 neurons, we perform numerical simulations of the model presented in Materials and Methods. Parameters of the model are fitted to known anatomical and physiological data as summarized in Table 1. Numerical simulations are performed for stimuli of varying size and contrast. In the figures, such stimuli are represented as gratings, to facilitate the link with experiments. However, in our network model, the size of a stimulus is simply taken to be the spatial extent (in degrees) of the feedforward afferents carrying nonzero currents to the V1 layer, and the contrast of the stimulus is taken to be the strength of these currents (see Materials and Methods).

Iso-orientation surround suppression in V1 has traditionally been attributed to intra-areal horizontal connections (Gilbert et al., 1996; Somers et al., 1998; Fitzpatrick, 2000). Because these connections terminate on both excitatory and inhibitory neurons (Martin and Whitteridge, 1984; Kisvárday et al., 1986; McGuire et al., 1991), surround suppression has been proposed to be gen- 


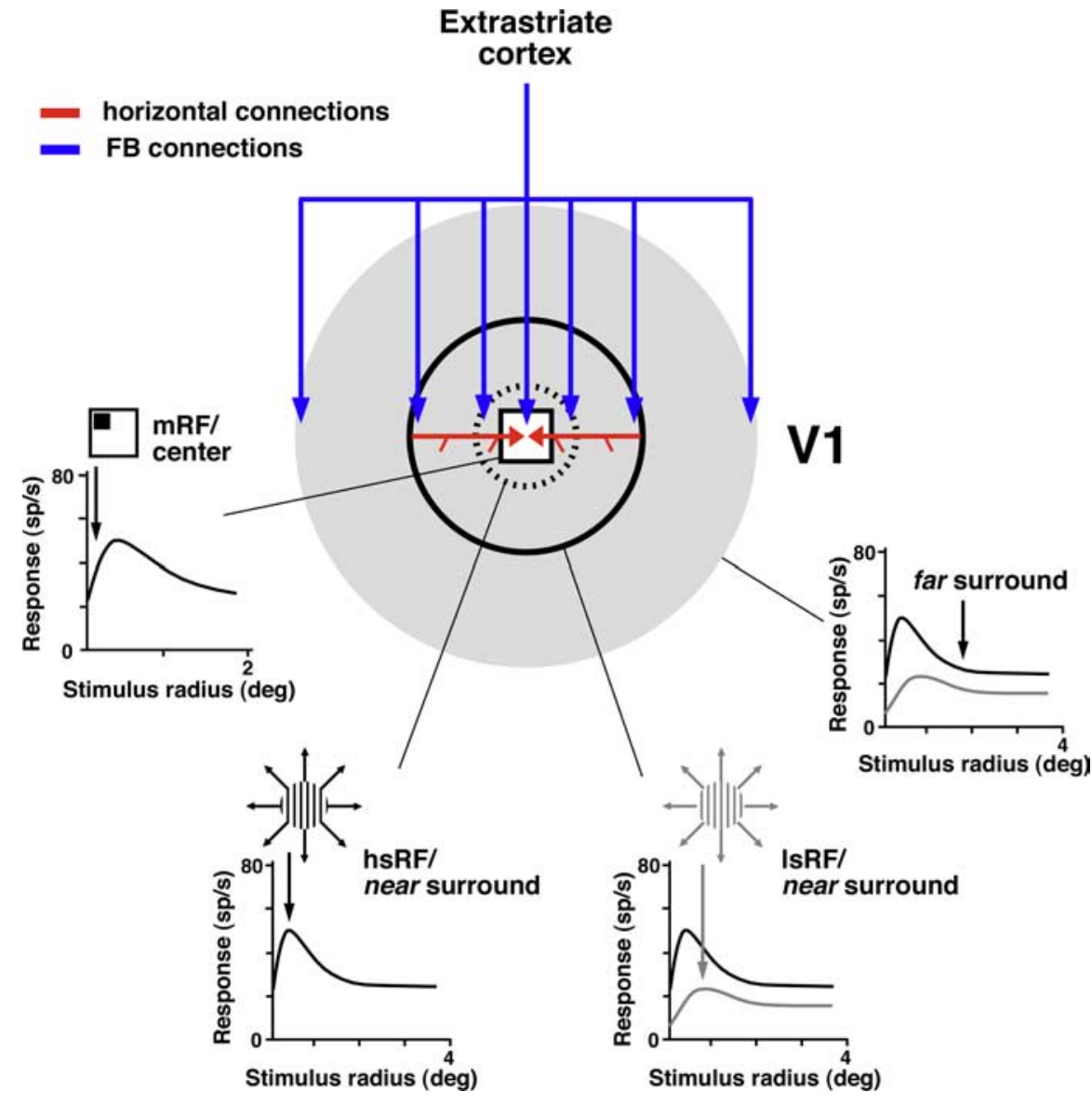

Figure 1. Different components of the RF center and surround of V1 neurons and their hypothesized anatomical substrates. In the center of the figure is a schematic diagram of the various components of the RF center and surround of an example V1 neuron; these are surrounded by four identical size-tuning curves for this neuron, over which an icon and arrow indicate the size of the respective RF component. Black and gray curves are size-tuning curves measured at high- and low-stimulus contrast, respectively. The white square area is the $\mathrm{mRF}$ or RF center; this is the RF region over which presentation of optimally oriented stimuli evokes spikes from the cell. Experimentally, the $\mathrm{mRF}$ is measured using small, high-contrast moving stimuli and delimiting the area where spikes are evoked from the cell (icon on the top-left size-tuning curve) (Hubel and Wiesel, 1962; Barlow et al., 1967); the arrow under the $\mathrm{mRF}$ icon indicates the size of the $\mathrm{mRF}$ relative to the peak of the high-contrast size-tuning curve. The dashed ring is the hsRF; this is measured by presenting high-contrast gratings of increasing radius (icon on the center-left size-tuning curve) and defined as the stimulus radius at the peak response of the neuron (arrow under the hsRF icon). The region between the mRF and the hsRF is the region over which presentation of high-contrast gratings at the same orientation as the center grating facilitates the response of the cell to optimally oriented gratings in the center. The hsRF radius is about twice that of the mRF. The continuous ring is the IsRF; this is measured and defined as the hsRF size, but using low-contrast gratings of increasing radius (icon on the center-right size-tuning curve). The region between the hsRF and the IsRF is the region over which presentation of gratings at the same orientation as the center grating suppresses or facilitates the response of the cell to optimally oriented gratings in the center, depending on the contrast of the grating. Note the shift to the right of the peak response at low contrast (Sceniak et al., 1999). Gray area, RF surround. We consider two separate regions of the surround depending on their proximity to the RF center: (1) the near surround is the region between the mRF and the ISRF, (2) the far surround is the region outside the IsRF over which presentation of stimuli at the same orientation as the center stimulus usually suppresses the response of the cell to optimally oriented gratings in the center. Intra-areal V1 horizontal connections (red) are commensurate with the ISRF size of their V1 neurons of origin, whereas extrastriate $F B$ (blue) connections to $V 1$ are commensurate with the full spatial scale of the center and surround field of 11 neurons (Angelucci et al., 2002b). Feedforward geniculocortical connections (data not shown) are commensurate with the hsRF size of their recipient V1 neurons (Angelucci and Sainsbury, 2006).

erated by lateral excitation of local inhibition (Lund et al., 1995; Somers et al., 1998). Thus, following previous recurrent network models (Somers et al., 1998; Dragoi and Sur, 2000), we assume that stimulation of the near surround modulates the response to a center stimulus via horizontal connections targeting both excitatory and inhibitory neurons in the center. The interneurons are assumed to have a higher threshold and gain than the local excitatory neurons whose output they control (Fig. 3b) (see Materials and Methods) and, thus, only generate suppression under suffi- ciently high levels of excitation, for example, when the contrast and/or size of a grating stimulus is sufficiently large. At low levels of excitation, such as for small or low-contrast stimuli, the inhibitors are inactive, and stimulation of the near surround facilitates the center response. The key novel features of our model are as follows: (1) FB from the extrastriate cortex provides an additional source of excitation to excitatory neurons in the center and in the near surround; (2) fast suppression from the far surround is assumed to occur via $\mathrm{FB}$, rather than via a cascade of horizontal connections. Our model provides a specific solution to the puzzle of how exclusively excitatory interareal $\mathrm{FB}$ connections, which target predominantly excitatory neurons in V1, can mediate far surround suppression of the center neurons; namely by targeting excitatory neurons in the near surround, which in turn, via horizontal connections, excite the local inhibitory neurons in the center. It also follows that if the local interneurons are inactive, then stimulation of the far surround is facilitatory. Therefore, our model also predicts that the "suppressive far surround" of V1 neurons is not always suppressive (see below, Contrast-dependent suppression and facilitation from the far surround: a model prediction).

Contrast-dependent spatial summation We first use our model to simulate experiments in which the RF size is measured as the stimulus radius at the peak response of an area-summation curve (the summation RF), using high- or low-contrast gratings. The summation RF size is known to depend on the stimulus contrast, with lower contrasts yielding larger summation RF sizes (Sceniak et al., 1999) (Fig. 1). The results of these simulations are shown in Figure $4 a-c$. Increasing the size of the stimulus leads to an increased response of the center excitatory neurons. This facilitation turns into suppression as the stimulus size is further increased, because the local interneurons, now sufficiently stimulated, start firing (Fig. 4a). The stimulus size at which the interneurons are activated, however, depends on the stimulus contrast. At lower contrast, the firing rates of all excitatory neurons are also lower, and so the interneuron has to integrate over a larger area to reach its firing threshold. This accounts for the contrast-dependence of the RF size measured via the area-summation curves. In order for the above mechanism to be effective, in the model, the center interneurons must be strongly driven by horizontal connections, more so than the center excitatory neurons (Fig. $4 b, c$ ). Figure $4, b$ and $c$, additionally shows how, in the model, the various input currents to the excitatory and inhibitory neurons vary with stimulus size. 
Another interesting feature of the curves shown in Figure $4 a$ is that there is an oscillatory component to the variation in response as a function of stimulus size. This is because of disinhibition of the center excitatory neurons caused by suppression of the excitatory neurons in the near surround driving the center inhibitory neurons. Such suppression in turn depends on the level of excitation of horizontal connections to near surround neurons arising from the center and far surround. This predicted disinhibition of the center excitatory neurons has been generally overlooked in the experimental data, partly because of the coarser sampling of the stimulus size used in most experiments compared with our computer simulations, and partly because it was considered as noise. However, this phenomenon can be observed in several experimental papers published previously (DeAngelis et al., 1994; Li and Li, 1994; Sengpiel et al., 1997; Walker et al., 2000), although in most cases the authors failed to appreciate it and, thus, did not comment about it. Motivated by this specific prediction of our model, in recent physiological studies we (Ichida et al., 2005) have observed such an oscillatory component in the size-tuning curve of a significant proportion of $\mathrm{V} 1$ neurons.

In Figure $4 d-f$, we show a sequence of diagrams for increasing stimulus size, highlighting the network components that are active and the major afferent pathways from the active regions to the center neurons. A stimulus fitted to the size of the hsRF activates the center and part of the near surround (Fig. 4d). The center receives excitatory inputs via feedforward afferents, via horizontal connections from the active region of the near surround, and via $\mathrm{FB}$ connections from extrastriate cortex. At high contrast, the local inhibitors are assumed to be close to threshold, so that an additional increase in stimulus size beyond the hsRF size, as in Figure 4e, leads to suppression of the center response. In contrast, expansion of a low-contrast stimulus beyond the hsRF size results in facilitation, until the full extent of the near surround is active (Fig. 4e). Finally, high- or lowcontrast stimuli that extend well beyond the lsRF activate the far surround (Fig. $4 f$ ). This suppresses the center via FB connections exciting neurons in the near surround, which then excite local interneurons in the center. In principle, in the model, it is possible for the far surround to suppress the center via a cascade of horizontal connections from the far, to the near surround, to the center (Fig. 2). Although such a cascade is too slow to account for the fast onset of suppression (Girard et al., 2001; Angelucci and Bullier, 2003; Bair et al., 2003) (see Introduction), it could contribute to the late phase of the suppression. In our simulations, such a contribution is negligible because of

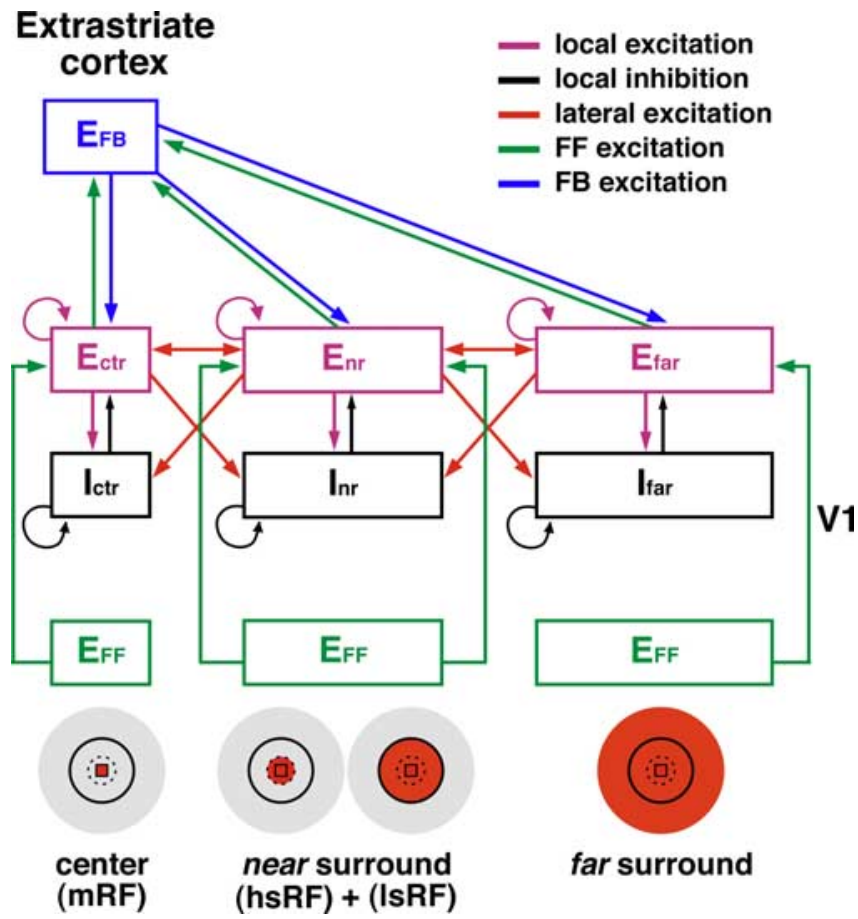

Figure 2. Basic architecture of the recurrent network model. Schematic diagram of the connections used in the network model. Different connection types are indicated as color-coded arrows. Purple and black boxes represent populations of excitatory (E) or inhibitory (I) V1 neurons, respectively, labeled according to the position of their RF center relative to that of the center neurons. Accordingly, ctr are the neurons in the RF center or mRF; nr are the neurons in the near surround, of which those closer to the center fall within the hsRF of the center neurons, whereas those located farther from the center fall within the IsRF of the center neurons; far are the neurons in the far surround. $\mathrm{E}_{\mathrm{FF}}$, Excitatory neurons in other V1 layers sending feedforward afferents to the Eneurons in V1 layers $2 / 3 . \mathrm{E}_{\mathrm{FB}}$, excitatory neurons in extrastriate cortex sending feedback projections to the Eneurons in V1. FB connections are spatially highly divergent and convergent. Note the absence of direct $F B$ inputs to I neurons. The latter receive monosynaptic inputs only from $V 1$ horizontal connections (red arrows) and from local $E$ neurons via local recurrent connections (purple arrows). Icons at the bottom represent the different components of the RF center and surround (same conventions as in Fig. 1), with red areas indicating RF components that are stimulated when each respective submodule is consecutively (from left to right) activated by a stimulus of increasing radius.
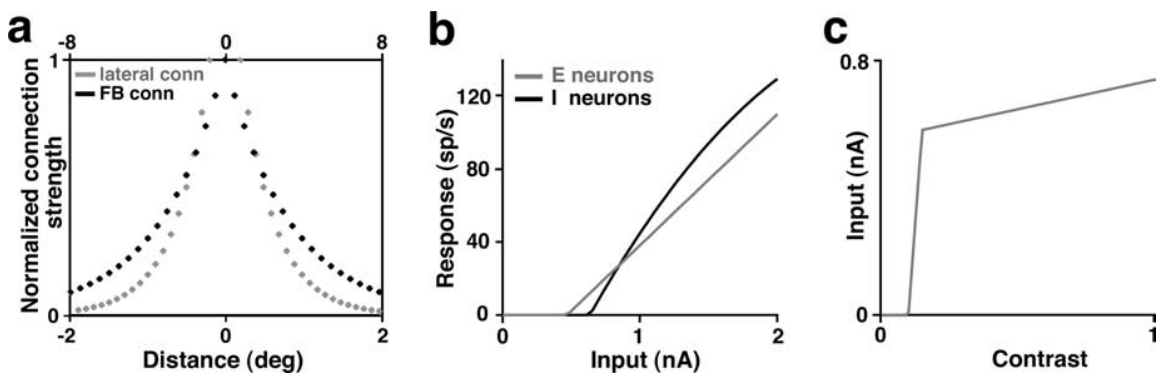

Figure 3. Functional specification of the network model. $\boldsymbol{a}$, Different spatial scales used in the model for the FB (black) and the lateral (or horizontal; gray) connections. Plotted is the normalized strength of lateral and FB connections to a postsynaptic neuron as a function of the distance of the RF centers of the presynaptic neurons from the RF center of the postsynaptic neuron. Note the different scales on the $x$ axes (the top one applies to the FB connections, the bottom to the lateral connections). $\boldsymbol{b}$, Firing rate of the V1 local excitatory (E; gray) and inhibitory (I; black) neurons in the model, plotted against the input current. $c$, Input current of the V1 excitatory neuron as a function of stimulus contrast.

surround suppression of excitatory V1 neurons at each step in the chain, which prevents horizontal propagation of signals. However, although in our model all excitatory neurons show surround suppression, in real V1 some neurons do not (Levitt and Lund, 2002). These cells could propagate signals horizontally and, thus, contribute to the late phase of the suppression.

Contrast-dependent suppression and facilitation from the far surround: a model prediction

To investigate more directly the contribution of FB connections to the far surround of V1 neurons, we simulate an experiment in 

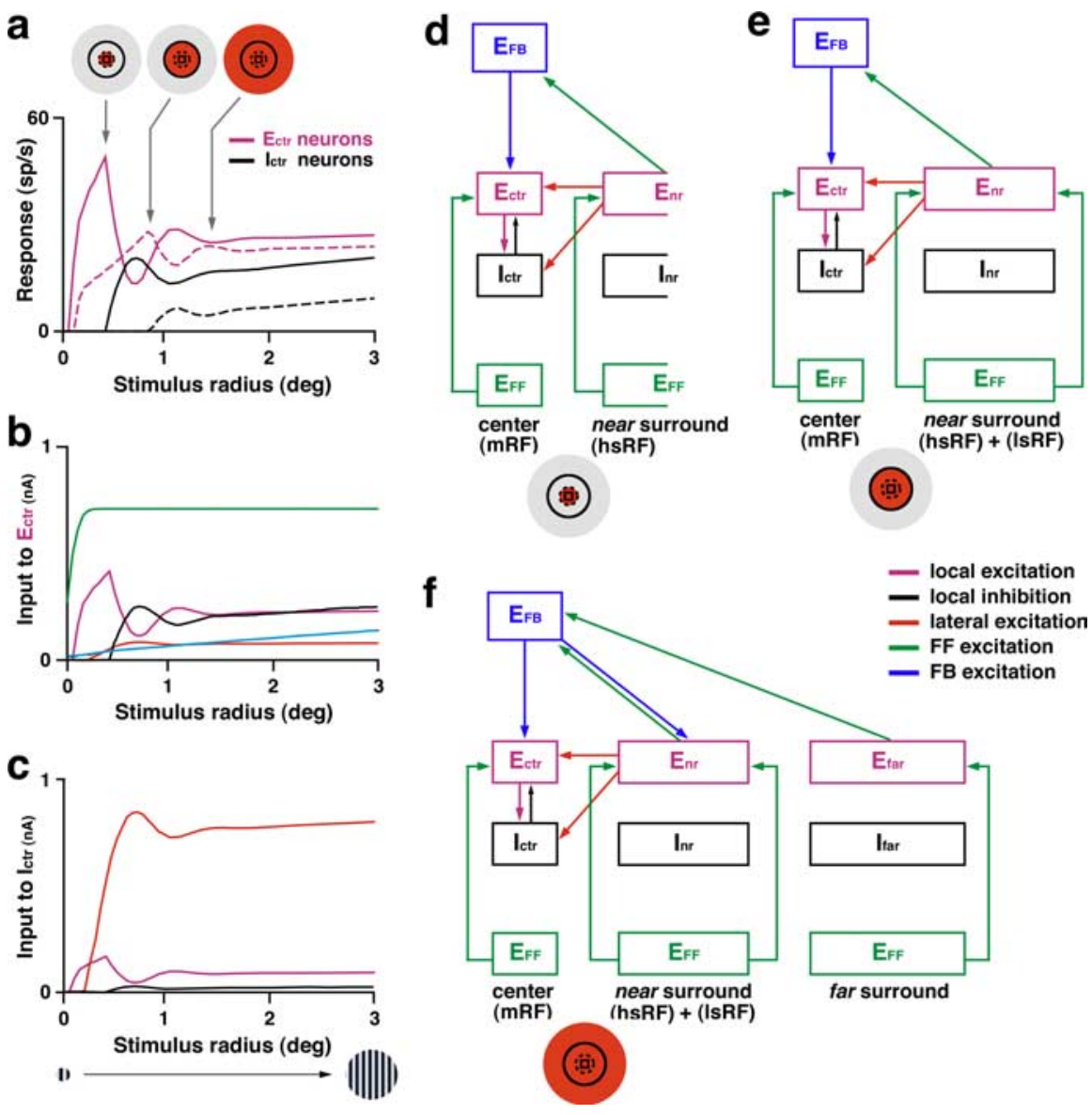

Figure 4. Contrast-dependent spatial summation in the network model. $\boldsymbol{a}$, Response (spikes/second) of the center excitatory ( $E_{c t r i}$ purple) and inhibitory $\left(I_{c t r} ;\right.$ black) neurons as a function of the size of the afferent stimulus (in degrees) for two different stimulus contrasts ( 85 and $15 \%$, solid and dashed lines, respectively). Icons at the top represent the different components of the $\mathrm{RF}$ center and surround (as in Figs. 1,2), with red areas indicating the RF regions that are activated at the indicated points in the size-tuning curve. These same icons are shown in $\boldsymbol{d}-\boldsymbol{f}$ to indicate the respective active network components and pathways. $\boldsymbol{b}, \boldsymbol{c}$, Input currents to the center excitatory neurons $(\boldsymbol{b})$ and to the center inhibitory neurons $(\boldsymbol{c})$ as a function of the afferent stimulus size (in degrees). The different input types are color coded according to the legend inf. Icons under the abscissa in c show the stimulus used and are valid for $\boldsymbol{a}-\boldsymbol{c} . \boldsymbol{d}-\boldsymbol{f}$, A sequence of diagrams highlighting, for increasing stimulus size, the network components that are active and the major afferent pathways from the active regions to the center neurons. Conventions are as in Figure 2.

which afferent stimulation of the region between the hsRF and lsRF is withdrawn. This allows us to minimize the afferent drive of horizontal connections in the near surround so as to unmask the effect of FB connections from the far surround. To achieve this, a stimulus analogous to a high-contrast annular grating is presented in the far surround together with a central highcontrast stimulus fitted to the size of the hsRF (stimulus shown in Fig. $5 b$ ). The inner radius of the "annulus" is systematically decreased from the far surround to a size no smaller than that of the lsRF, so that near surround neurons in the lsRF but beyond the hsRF never receive afferent stimulation (Fig. $5 a, b$ ). As the inner radius is decreased, more neurons in the far surround receive afferent stimulation. This leads to suppression of the excitatory neurons in the center (Fig. $5 a$ ), as observed experimentally (Levitt and Lund, 2002). The active components in the network, and the main pathways relaying information to the center neurons in response to this stimulus configuration are illustrated in Figure $5 e$. The mechanism leading to the response suppression is the same as that illustrated in Figure 4. Specifically, as more neurons in the far surround are activated, excitatory neurons in the near surround increase their firing rate (Fig. 5b); this is caused by increased excitation received via $\mathrm{FB}$ connections activated by stimulation of the far surround. The near surround neurons, then, activate the center interneurons via horizontal connections (Fig. 5a,e), resulting in suppression. However, our model also demonstrates that, when a low-contrast central stimulus is presented together with a high-contrast annulus in the far surround (stimulus shown in Fig. $5 d$ ), decreasing the inner radius of the annulus (i.e., stimulating an increasingly larger number of neurons in the far surround), can lead to initial facilitation of the center excitatory neurons followed by suppression (Fig. $5 c-d$ ). This is attributable to the fact that the lowcontrast central stimulus is too weak to activate the center interneurons by itself (Fig. 5c); thus, stimulation of the far surround initially facilitates the response of the center neurons, because FB inputs to the center sum with afferent and horizontal inputs, until a critical annulus size is reached, beyond which the interneurons are activated (Fig. $5 c$ ) and suppression occurs. It is important to emphasize that polysynaptic chains of horizontal connections are unlikely to contribute to the modulatory effects of the far surround in these "annular grating" experiments. This is because the excitatory neurons whose RFs lie in the visual field location of the blank stimulus do not receive afferent drive and, thus, cannot effectively relay signals to their postsynaptic V1 neurons because of the purely modulatory (not driving) effect of lateral connections to excitatory neurons (Hirsch and Gilbert, 1991).

One of the advantages of using a recurrent network model is that it allows us to make predictions regarding the population activity profile of neurons across V1 in response to visual stimuli. Figure $6 a$ shows the predicted activity profile of excitatory neurons as a function of position relative to the center neuron population, in the case of a small highcontrast stimulus $\left(0.4^{\circ}\right.$ radius; i.e., the size of the hsRF, as derived from Fig. $4 a$ ) and a large high-contrast stimulus ( $1.5^{\circ}$ radius; i.e., extending into the far surround, as derived from Fig. $4 a$ ). The responses of the neurons in the center and near surround decrease with increasing stimulus size (thin vs thick line), as expected from the simulated area summation experiments (Fig. $4 a$ ). However, the responses of neurons with their RF centers located at the border of the large stimulus, in the far surround, are not suppressed. Figure $6 b$ shows the predicted activity profile for a small high-contrast central stimulus $\left(0.5^{\circ}\right.$ radius; i.e., just larger than the hsRF of the neurons, but smaller than the lsRF, as derived from Fig. $4 a$ ) presented with and without a high-contrast annular stimulus $\left(3.2^{\circ}\right.$ inner radius and $8^{\circ}$ outer radius) in the far surround. When the annulus is presented together with the central stimulus, the responses of the center excitatory neurons are suppressed, whereas the responses of the excitatory neurons in the near surround are facilitated. This can be explained by the same mechanism illustrated in Figure 5, $a$ and $b$, namely, that activation of $\mathrm{FB}$, because of stimulation of the far surround, en- 


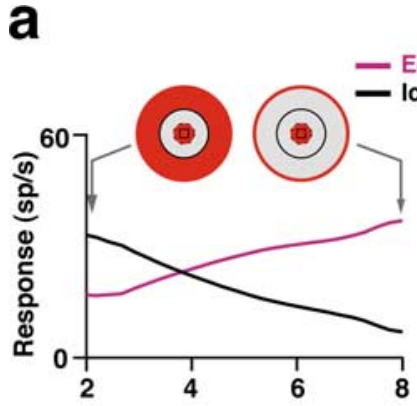

C

Ectr neurons

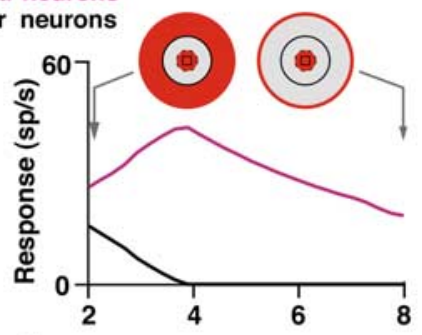

b

d
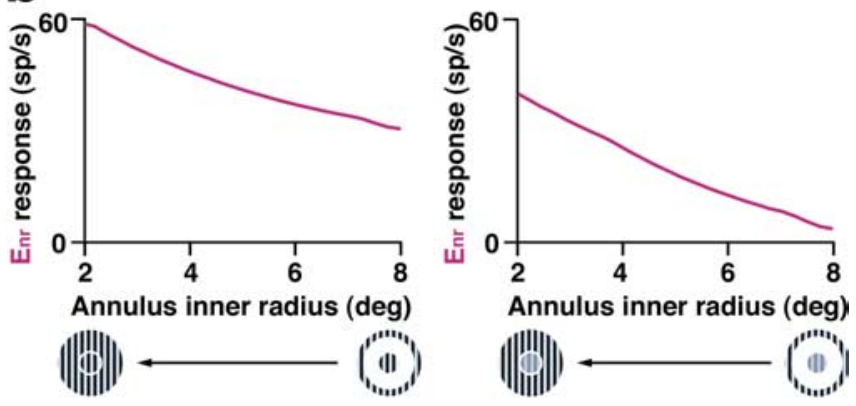

e

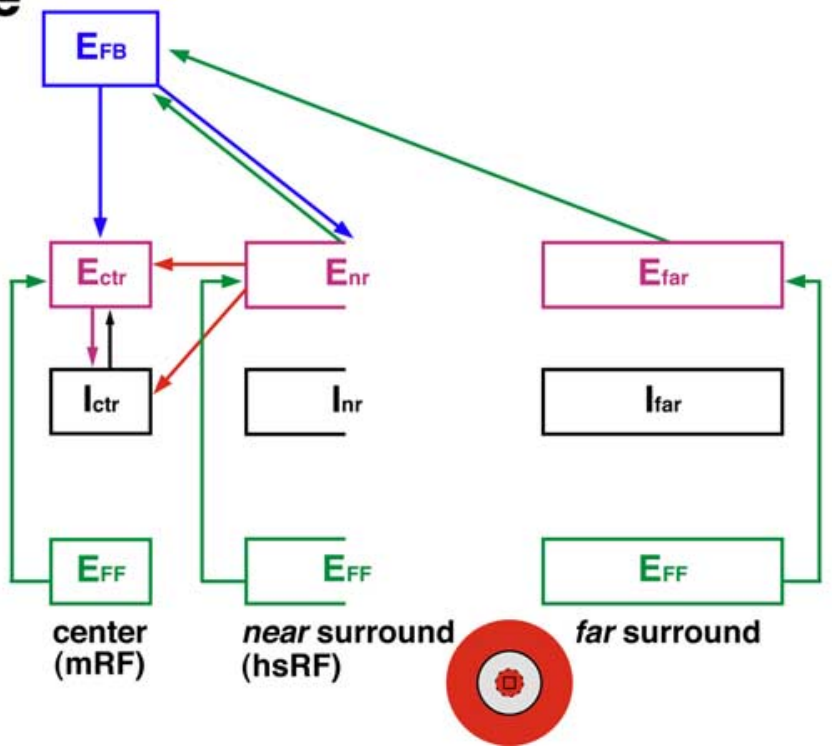

Figure 5. Contrast-dependent suppression and facilitation from the far surround, in the network model. $\boldsymbol{a}$, Response (spikes/second) of the center excitatory ( $E_{c t r i}$ purple) and inhibitory (I $I_{\text {ctr }}$ black) neurons to a high-contrast $(85 \%)$ central stimulus of $0.5^{\circ}$ radius (i.e., just larger than the hsRF of the neuron; Fig. 4a) plotted against the inner radius of a high-contrast (85\%) annular stimulus of an $8^{\circ}$ outer radius presented together with the central stimulus (stimulus configuration shown in $\boldsymbol{b}$ ). Other conventions are as in Figure 4. $\boldsymbol{b}$, Same as in $\boldsymbol{a}$, but for excitatory neurons in the near surround $\left(E_{n r}\right)$. Icon under the abscissa represents the stimulus used in $\boldsymbol{a}$ and $\boldsymbol{b}$. c, Same as in $\boldsymbol{a}$ but the configuration of the stimulus used (shown in $\boldsymbol{d}$ ) consists of a low-contrast (15\%) central stimulus ( $0.5^{\circ}$ radius) presented together with a high-contrast $(85 \%)$ annular stimulus in the far surround. $\boldsymbol{d}$, Same as in $\boldsymbol{c}$, but for excitatory neurons in the near surround $\left(E_{n r}\right)$. I con under the abscissa represents the stimulus used in c and $d$. $e$, Diagram highlighting, for the RF regions activated by the stimulus (red areas in the icon at the bottom), the network components that are active and the major afferent pathways from the active regions to the center neurons. Conventions are as in Figures 2 and $4 d-f$.

hances the response of excitatory neurons in both the center and near surround, which in turn activate the center inhibitors (Fig. $5 e$ ). It is worth emphasizing that the excitatory neurons in the near surround are not suppressed, but are facilitated by the far

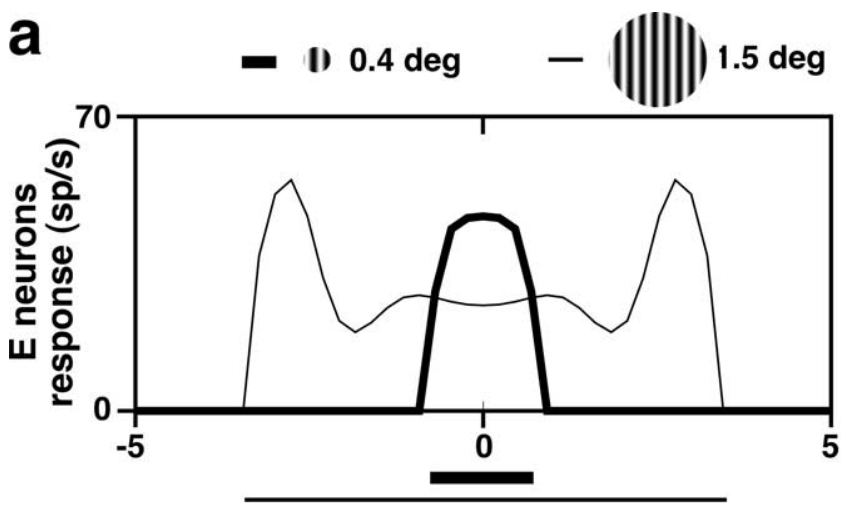

Position in cortex $(\mathrm{mm})$

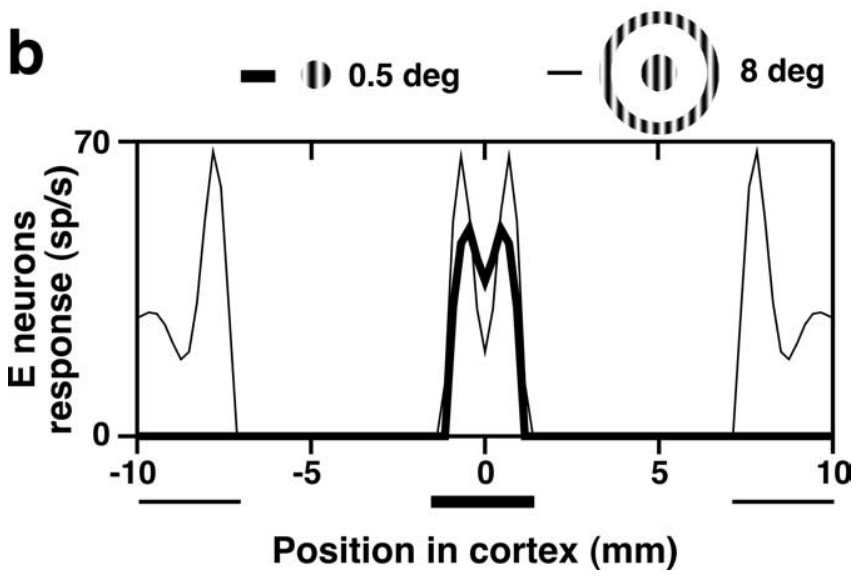

Figure 6. Population activity profile of the excitatory neurons across V1. $a$, Activity profile for a small ( $0.4^{\circ}$ radius; thick line) and a large ( $1.5^{\circ}$ radius; thin line) high-contrast $(85 \%)$ stimulus. Plotted are the firing rates (spikes/second) of the excitatory neurons against their location in V1. $\boldsymbol{b}$, Activity profile for a small ( $0.5^{\circ}$ radius) high-contrast $(85 \%)$ central stimulus presented alone (thick line) or together (thin line) with a high-contrast $(85 \%)$ annular stimulus ( 3.2 and $8^{\circ}$ inner and outer radius, respectively). The cortical location of the neurons were computed by converting the distance in degrees between their RF centers into cortical distance, using a cortical magnification factor of $2.3 \mathrm{~mm} /{ }^{\circ}$ (see Materials and Methods).

surround stimulus; this occurs because their local inhibitors, in contrast to the local inhibitors in the center, do not receive sufficiently high afferent drive because of the small size of the central stimulus. Although this modulation of the cortical response profile predicted by our model has not been investigated experimentally, higher neuronal responses at the borders of stimuli compared with their centers have been observed previously in experimental data using figure-like stimuli defined by texture (Lee et al., 1998) or color (Friedman et al., 2003).

\section{Inactivation of the feedback pathway}

Reversible inactivation of the extrastriate cortex had previously revealed a facilitatory influence of $\mathrm{FB}$ connections on the RF center response of V1 neurons (Sandell and Schiller, 1982; Mignard and Malpeli, 1991; Hupé et al., 1998). The question of how interareal FB connections contribute to suppressive contextual effects was directly addressed in a series of experiments by Bullier and coworkers using reversible inactivation of extrastriate cortex (Hupé et al., 1998; Bullier et al., 2001). Inactivation of FB connections arising from area MT greatly reduced the suppressive effect of surround stimulation onto the center responses of neurons in areas V1, V2, and V3 (in area V3, it sometimes even 
caused facilitation). The largest reduction in surround suppression was observed for low-saliency stimuli.

In our network model, we can mimic the effect of FB inactivation simply by setting the values for the weights of the interareal $\mathrm{FB}$ connections to zero. To determine the contribution of FB connections to the center and surround of V1 neurons, we consider the change in response of the center excitatory neurons to a central stimulus presented alone or together with a surround stimulus, with and without active FB connections, and for centersurround stimuli with different saliencies. As in the experimental studies (Hupé et al., 1998; Bullier et al., 2001), here we define the "response change" as follows:

$$
-100 \times \frac{R_{C}-R_{C S}}{R_{C}},
$$

where $R_{C}$ and $R_{C S}$ are the responses of a neuron to the presentation of the central stimulus alone and of the central stimulus shown together with a surround stimulus, respectively. Stimulus saliency here is defined as $C_{\text {center }} / C_{\text {surround }}$, where $C_{\text {center }}$ and $C_{\text {surround }}$ are the contrasts of the central and surround stimuli, respectively. To obtain stimuli with different saliency, the surround stimulus is always presented at low contrast (15\%), whereas the contrast of the central stimulus $(>15 \%)$ is changed. Note that the central stimulus is fit to the optimal RF size of the neurons whose response we wish to determine; this is defined as the stimulus size at peak response, derived from the size-tuning curve of the neurons, and varies with the contrast of the stimulus (Fig. 4a). Thus, for stimuli of different saliency, the central stimulus ranges in size from the hsRF to the lsRF, and therefore also includes part of, or all of, the near surround. Figure 7, $a$ and $b$, shows the response of the center neurons to a central stimulus presented either alone or together with a surround stimulus. The response with and without FB is compared under low- (Fig. 7a) and high-saliency (Fig. $7 b$ ) conditions. In the case of the central stimulus alone, removal of FB leads to a reduction in the response of the center neurons, as in the experimental findings (Sandell and Schiller, 1982; Mignard and Malpeli, 1991; Hupé et al., 1998; Bullier et al., 2001). In our model, this follows from the fact that, under normal conditions, FB excites excitatory neurons in the center (Fig. 2). In the presence of FB connections, the response of the center neurons to the central plus surround stimulus is suppressed relative to the response to the central stimulus alone, and the degree of suppression is greater for high-saliency stimuli. In the latter case, the amount of suppression is similar with and without FB (Fig. 7b). In contrast, for low-saliency stimuli, there is a significant reduction in the suppression when $\mathrm{FB}$ is removed (Fig. 7a). The latter result is consistent with the experimental findings in V1 after inactivation of FB from MT (Bullier et al., 2001). Note that the effects of FB inactivation on the responses of V1 neurons are quantitatively different from those observed by the same experimenters in V3 neurons (Hupé et al., 1998). Figure $7 c$ shows the response change (as defined above) as a function of the stimulus saliency, with and without FB connections. The reduction in surround suppression when inactivating the $\mathrm{FB}$ is clearly strongest for low-saliency stimuli. In our model, the reduction in suppression is caused by the fact that the suppressive effect of the far surround is mediated by FB connections targeting excitatory neurons in the near surround (Fig. 2). Removal of FB reduces excitation of near surround neurons and, consequently, of the center local inhibitors that they contact. The results shown in Figure $7 c$ are qualitatively consistent with the experimental findings in V1 (Bullier et al., 2001), and suggest a crucial role for
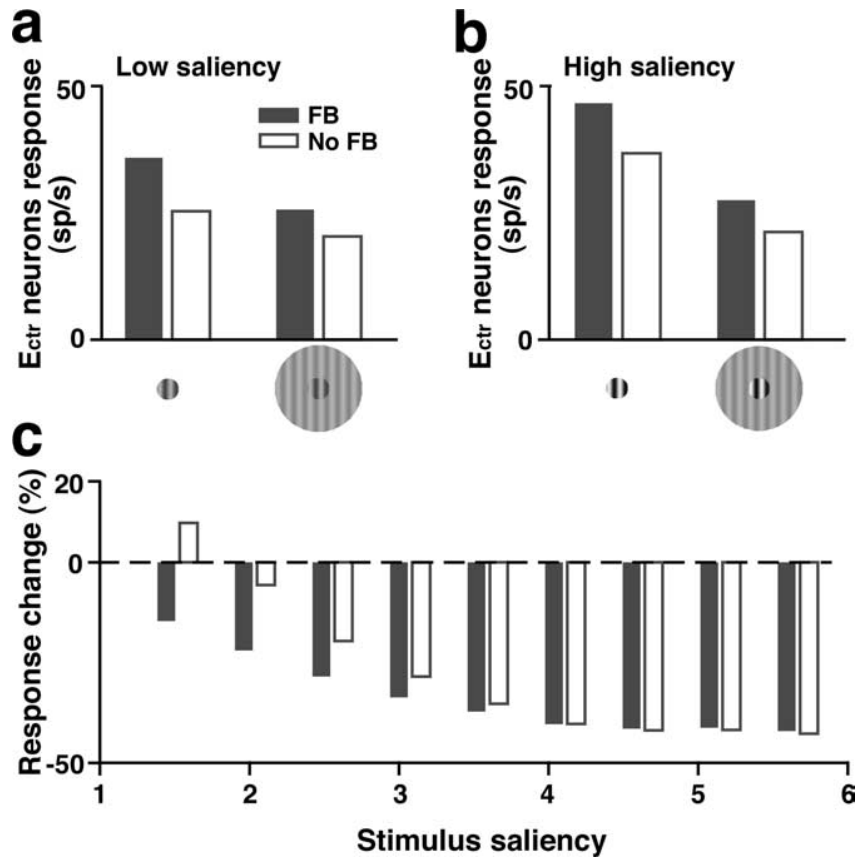

Figure 7. Center-surround interactions with and without feedback, in the network model. $\boldsymbol{a}$, Response (spikes/second) of the center excitatory neurons $\left(E_{c t r}\right)$ to the central stimulus alone (small grating; $0.68^{\circ}$ radius), and to the central stimulus presented together with a surround stimulus (large grating; surround stimulus of $0.68^{\circ}$ inner radius and $8^{\circ}$ outer radius) in the presence of FB (gray bars) or with the FB connections inactivated (white bars). The center plus surround stimulus was presented at low saliency (38 vs 15\% contrast; see Results for definition of saliency). $\boldsymbol{b}$, same as in $\boldsymbol{a}$ but for a high-saliency center plus surround stimulus (77 vs $15 \%$ contrast). The size of the central stimulus was always fitted to the stimulus size at the peak response of the size-tuning curve (i.e., the optimal size), which varies with the stimulus contrast; thus, in $\boldsymbol{b}$, the central stimulus had a radius of $0.49^{\circ}$, which is the optimal stimulus size at $77 \%$ contrast. c, Response change (see Results for definition) of the center excitatory neurons as a function of stimulus saliency, with and without FB. For each contrast, the central stimulus was presented at the optimal size.

interareal FB connections in surround suppression and, possibly, figure-ground segregation. Our model predictions, however, differ quantitatively from the data of Bullier et al. (2001), in that the predicted reduction in surround suppression is restricted to saliency values ranging between 1 and 6, which are defined by Bullier et al. (2001) as "low saliency" (stimuli with saliency values $>15$ were defined by these authors as "high saliency"). This quantitative difference between the actual data and our model is likely attributable to the fact that, whereas Bullier et al. (2001) used single bar stimuli in the RF center to drive the cells, in our model we use the equivalent of gratings stimuli optimized for size at each contrast value (i.e., the size of the hsRF at high contrast, and the lsRF size at low contrast). Thus, our stimuli would drive V1 cells much more vigorously than single bars. In other words, "high-saliency" bar stimuli used in the experiments are likely to drive V1 neurons much like the lower saliency gratings used in our model, and therefore would push the saliency-response curve of Figure $7 c$ toward higher saliencies.

\section{Dynamics of contextual effects}

Recently, the short-term dynamics of suppressive contextual effects has been investigated in macaque V1 (Bair et al., 2003). The latency of suppression induced by stimuli in the far surround was found to be almost as short as the latency of suppression induced by stimuli in the near surround. The short delay in the onset of suppression induced by far surround stimulation is not consis- 
a
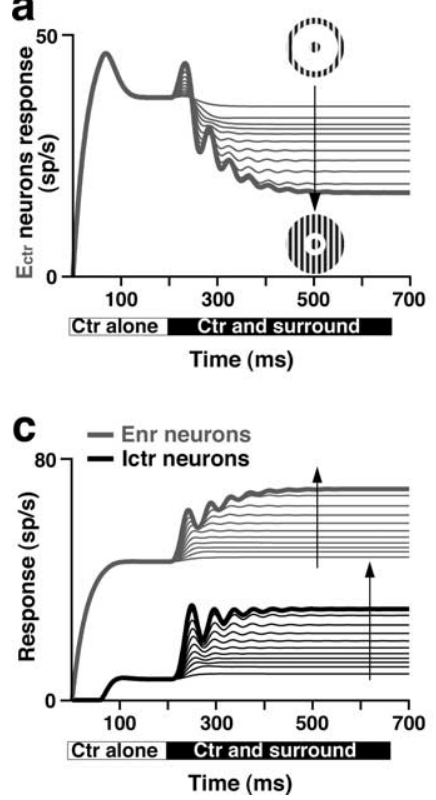

b
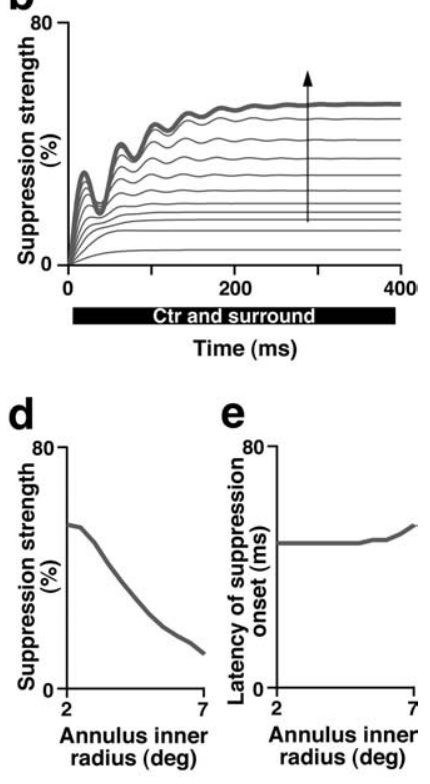

Figure 8. Dynamics and latency of surround suppression in the network model. $\boldsymbol{a}$, Response (spikes/second) of the center excitatory neurons $\left(E_{c t r}\right)$ to a high-contrast (85\%) central stimulus $\left(0.5^{\circ}\right.$ radius) first presented alone and, after $200 \mathrm{~ms}$, together with a high-contrast annular stimulus in the far surround of varying inner radius $\left(2.5-7^{\circ}\right)$, plotted against the time from the onset of the central stimulus. In $\boldsymbol{a}-\boldsymbol{c}$, the arrow points in the direction of the decreasing inner radius of the annulus, as indicated by the stimulus icon. $\boldsymbol{b}$, Suppression strength of the response of the center neurons induced by a stimulus in the far surround, plotted against the time from the onset of the suppression. Suppression strength is defined as $100^{*}\left(R_{C}-R_{C S} / R_{C}\right)$, where $R_{C}$ and $R_{C S}$ are the response of the neurons to the presentation of the central stimulus alone and of the central stimulus shown together with the surround stimulus, respectively. c, Responses (spikes/second) of inhibitory neurons in the center (black) and excitatory neurons in the near surround (gray) for annuli of varying inner radius. $\boldsymbol{d}$, Suppression strength of the steady-state response of the center excitatory neurons as a function of the inner radius of the surround annulus. $\boldsymbol{e}$, Latency of suppression onset (defined as the time from the onset of the annulus at which suppression strength reached $3 \%$ ) as a function of the inner radius of the surround annulus.

tent with polysynaptic chains of horizontal connections mediating this effect because of their slow conduction velocity. These results suggest a role for $\mathrm{FB}$ connections in the generation of suppression from the far surround.

We simulate these experiments and investigate the model's predictions for these delays. Figure $8 a$ shows the response, and Figure $8 b$ its suppression strength, of the center excitatory neurons to a stimulus protocol where first only an optimal central stimulus, the size of the hsRF, is presented, and then a highcontrast annular stimulus is flashed in the far surround, after the center response reaches its steady state $(>200 \mathrm{~ms})$. The response suppression is strongest for "annuli" with smaller inner radius (i.e., simulating larger regions of the surround) (Fig. 8d). In our model, the suppression from the far surround is mediated via relaying excitation from $\mathrm{V} 1$ neurons in the far surround to the extrastriate area (via feedforward connections), and from there to the excitatory neurons in the near surround (via FB connections), which in turn excite the center inhibitory neurons (via horizontal connections), as in Figure $5 e$. The predicted responses of excitatory neurons in the near surround and of the local inhibitory neurons in the center are shown in Figure $8 c$. The dynamics of the response of the inhibitory neurons in the center follows the response dynamics of the excitatory neurons in the near surround, which drive these inhibitory neurons. Importantly, the latency of suppression onset in the response of the center neurons is almost independent of the inner radius of the annulus (i.e., it is only weakly dependent on the location of stimulation in the surround) (Fig. 8e), as observed experimentally (Bair et al., 2003). In the model, this is because of the suppression being mediated via fast interareal FB connections, which introduce a delay of only a few milliseconds.

\section{Discussion}

We have developed an anatomically and physiologically constrained recurrent network model of center-surround interactions in macaque V1. In addition to V1 horizontal connections typically thought to underlie the extraclassical RF of V1 cells, our model includes interareal feedback projections based on recent data implicating feedback in center-surround interactions (for review, see Angelucci and Bullier, 2003; Shapley, 2004; Angelucci and Bressloff, 2006). We have used our model to elucidate the relative roles of horizontal and $\mathrm{FB}$ connections in generating the spatial summation properties of V1 neurons, their dependence on stimulus contrast, and the timing and dynamics of centersurround interactions. Our results support the hypothesis that extrastriate $\mathrm{FB}$ connections underlie the modulation of responses of V1 neurons arising from the far RF surround. We have proposed a solution to the puzzle of how excitatory FB connections, targeting almost exclusively excitatory neurons in V1, can generate far surround suppression, namely by targeting excitatory neurons in the near surround sending monosynaptic horizontal connections to inhibitory neurons in the RF center. While the current debate is centered on whether horizontal or feedback connections mediate center-surround effects, our model provides insights into how these two sets of connections interact to generate responses both within and outside the classical RF. Feedback-mediated iso-orientation surround suppression is also consistent with evidence in primates that FB axons link cortical territories of broadly similar orientation preference (Angelucci et al., 2003; Shmuel et al., 2005) (but see Stettler et al., 2002).

Our model can account for a wide range of physiological data in V1, including (1) contrast-dependent spatial summation (Sceniak et al., 1999), (2) size tuning (Sceniak et al., 2001; Cavanaugh et al., 2002a; Levitt and Lund, 2002), (3) far surround suppression seen in experiments in which the afferent drive to near surround neurons is partially withdrawn by interposing a blank between the grating in the RF center and that in the far surround (Levitt and Lund, 2002), (4) the reduction of RF center response and of surround suppression after inactivation of $\mathrm{FB}$ connections (Hupé et al., 1998; Bullier et al., 2001), and (5) the lack of dependence of the latency of surround suppression on the distance of the surround stimulus from the RF center (Bair et al., 2003).

As in a previous recurrent network model of center-surround interactions (Somers et al., 1998), as first suggested by Lund et al. (1995), we assume that stimulation of the near surround modulates the response to a center stimulus via horizontal axons targeting both excitatory and inhibitory neurons in the center, the latter having higher threshold and gain than the local excitatory neurons. The specific sign of the modulatory effect thus depends on the overall level of excitation reaching the local inhibitory and excitatory neurons, with facilitation predominating for low levels of excitation and suppression for high levels of excitation. Whereas the latter phenomenon has been observed in electrical stimulation experiments in slices of visual cortex (Hirsch and Gilbert, 1991; Weliky et al., 1995; Shao and Burkhalter, 1996), the specific mechanism that we have proposed for its implementation (i.e., the requirement for higher threshold and gain inhibi- 
tory neurons) represents a specific model prediction partially supported by experimental data (see also Materials and Methods). In cortical layer $2 / 3$, high gain interneurons with facilitating synapses have been found whose response can only be recruited in the late phase of the action potential train of their presynaptic pyramidal cells (Pouille and Scanziani, 2004; Kapfer et al., 2005). While higher threshold interneurons are an experimentally verifiable hypothesis of our model, these facilitating interneurons could offer an alternative mechanism to implement the delayed recruitment of inhibition that is central to our model. An additional modeling assumption not yet demonstrated experimentally, but not inconsistent with current data, is that these facilitating interneurons are driven by horizontal connections, and more strongly so than local excitatory neurons. This is what allows surround suppression rather than facilitation to occur at high contrast.

Our model extends the "lateral model" (Somers et al., 1998) by incorporating FB inputs from extrastriate cortex as an additional source of excitation to excitatory neurons in the center and in the near surround. The addition of FB connections allows our model to account for several aspects of center-surround interactions that the lateral model could not account for, namely (1) the lack of dependence of the latency of surround suppression on the distance of the surround stimulus from the RF center (Bair et al., 2003), (2) the effects of FB inactivation on V1 neurons responses to stimuli in their RF center (Sandell and Schiller, 1982; Mignard and Malpeli, 1991; Bullier et al., 2001) and on their modulation by stimuli in the surround (Bullier et al., 2001), (3) far surround suppression (Sceniak et al., 2001; Cavanaugh et al., 2002a; Levitt and Lund, 2002), and (4) far surround facilitation (see below).

A novel prediction of our model is that the far surround of $\mathrm{V} 1$ neurons is not always suppressive, but can facilitate the RF center response when the amount of excitatory input to local inhibitors is relatively low (e.g., when the center neurons are stimulated with a low-contrast stimulus in the presence of a stimulus in the far surround). Facilitation of the center response by iso-oriented stimuli in the surround has been reported typically when the surround region near the RF center is stimulated (Kapadia et al., 1995; Polat et al., 1998; Chisum et al., 2003). Iso-oriented stimuli in the RF center and far surround, instead, have typically been shown to be suppressive (DeAngelis et al., 1994; Levitt and Lund, 1997, 2002; Sengpiel et al., 1998; Sceniak et al., 2001; Cavanaugh et al., 2002b). Iso-orientation far surround facilitation, as predicted by our model, and as confirmed by our recent physiological studies in macaque V1 (Ichida et al., 2005), is inconsistent with difference (or ratio) of Gaussians descriptions of contextual effects in V1 (Sceniak et al., 2001; Cavanaugh et al., 2002a).

Our model nicely captures the finding of Bair et al. (2003) that the onset latency of surround suppression is nearly independent of the distance of the surround stimulus from the RF center. However, it cannot account for the finding, from the same study, that suppression from the far, but not the near, surround is predominantly transient. Possibly, extending the model to include mechanisms affecting the short-term dynamics of centersurround interactions, such as firing rate adaptation, could reveal transient suppression emerging from the network dynamics.

Our model relies on intracortical inhibition to generate surround suppression, and does not include a feedforward component that may contribute to the suppression. Recent data (Webb et al., 2005) suggest that surround suppression in primate V1 is likely generated by two concurrently operating mechanisms, an intracortical mechanism that is stimulus tuned and binocularly driven, and an untuned and monocularly driven mechanism that may result from the nonorientation-specific surround suppression of LGN afferents (Solomon et al., 2002). Although we plan to explore the contribution of feedforward mechanisms to centersurround interactions in V1, in this study we wished to determine the contribution of the intracortical component alone.

A recent study in cat $\mathrm{V} 1$ concluded, on the basis of inactivation of intracortical inhibition by $\mathrm{GABA}_{\mathrm{A}}$ receptor antagonists, that surround suppression in $\mathrm{V} 1$ does not depend on intracortical inhibition, but results from surround suppression of LGN afferents (Ozeki et al., 2004). Because our model relies on intracortical inhibition, it cannot easily account for this finding. However, although a feedforward component is likely to contribute to surround suppression in V1 (see above), it is difficult to fully reconcile this finding in cat with strong experimental evidence in primates for an intracortical origin of center-surround interactions. Specifically, although surround suppression in V1 is orientationtuned and mediated dichoptically (DeAngelis et al., 1992; Sengpiel et al., 1997; Webb et al., 2005), it is not orientation-tuned in the LGN (Solomon et al., 2002; Bonin et al., 2005) (but see Sillito et al., 1993). Furthermore, geniculocortical afferents in macaque are not sufficiently extensive to account for the much larger surround fields of V1 neurons (Angelucci and Sainsbury, 2006). It is possible the GABA block performed by these authors was not sufficiently complete to abolish surround suppression.

An alternative mechanism to intracortical inhibition that combines the results of Ozeki et al. (2004) with evidence for an intracortical origin of surround suppression is withdrawal of afferent excitation (resulting from surround suppression of LGN afferents) to the cortical center neuron and to neurons sending horizontal and FB connections to the center. In this scenario, our model architecture (Fig. 2) would still be valid, but withdrawal of excitation would replace intracortical inhibition. Withdrawal of afferent excitation to horizontal and FB neurons projecting to the center neuron could also account for the orientation selectivity of center-surround interactions, which would result from the orientation-specificity of intracortical connections. However, although withdrawal of afferent excitation combined with intracortical interactions could generate the surround suppression seen in the spatial summation results of Figure $4 a$, it is difficult to envision how such a mechanism could also account for far surround suppression at high contrast seen in the annulus experiments of Figure $5 a$. In the latter, the surround stimulus is located far beyond the spatial extent of feedforward connections to the center and thus cannot directly affect the response of LGN afferents to the RF center; a more plausible explanation is that the far surround stimulus suppresses the response of the center via intracortical feedback.

\section{References}

Albright TD, Desimone R (1987) Local precision of visuotopic organization in the middle temporal area (MT) of the macaque. Exp Brain Res 65:582-592.

Allman J, Miezin F, Mc Guinness E (1985) Stimulus specific responses from beyond the classical receptive field: neurophysiological mechanisms for local-global comparisons in visual neurons. Annu Rev Neurosci 8:407-430.

Angelucci A, Bressloff PC (2006) Contribution of feedforward, lateral and feedback connections to the classical receptive field center and extraclassical receptive field surround of primate V1 neurons. Prog Brain Res 154:93-121.

Angelucci A, Bullier J (2003) Reaching beyond the classical receptive field of V1 neurons: horizontal or feedback axons? J Physiol (Paris) 97:141-154. Angelucci A, Sainsbury K (2006) Contribution of feedforward thalamic afferents and corticogeniculate feedback to the spatial summation area of macaque V1 and LGN. J Comp Neurol 498:330-351. 
Angelucci A, Levitt JB, Lund JS (2002a) Anatomical origins of the classical receptive field and modulatory surround field of single neurons in macaque visual cortical area V1. Prog Brain Res 136:373-388.

Angelucci A, Levitt JB, Walton E, Hupé JM, Bullier J, Lund JS (2002b) Circuits for local and global signal integration in primary visual cortex. J Neurosci 22:8633-8646.

Angelucci A, Schiessl I, Nowak L, McLoughlin N (2003) Functional specificity of feedforward and feedback connections between primate V1 and V2. Soc Neurosci Abstr 29:911.2.

Bair W, Cavanaugh JR, Movshon JA (2003) Time course and time-distance relationships for surround suppression in macaque V1 neurons. J Neurosci 23:7690-7701.

Barlow HB, Blakemore C, Pettigrew JD (1967) The neural mechanisms of binocular depth discrimination. J Physiol (Lond) 193:327-342.

Bauer U, Scholz M, Levitt JB, Lund JS, Obermayer K (1999) A model for the depth dependence of receptive field size and contrast sensitivity of cells in layer 4C of macaque striate cortex. Vision Res 39:613-629.

Ben-Yishai R, Bar-Or RL, Sompolinsky H (1995) Theory of orientation tuning in visual cortex. Proc Natl Acad Sci USA 92:3844-3848.

Blakemore C, Tobin EA (1972) Lateral inhibition between orientation detectors in the cat's visual cortex. Exp Brain Res 15:439-440.

Bonin V, Mante V, Carandini M (2005) The suppressive field of neurons in lateral geniculate nucleus. J Neurosci 25:10844-10856.

Bosking WH, Zhang Y, Schofield B, Fitzpatrick D (1997) Orientation selectivity and the arrangement of horizontal connections in tree shrew striate cortex. J Neurosci 17:2112-2127.

Bressloff PC, Cowan JD (2002) An amplitude equation approach to contextual effects in visual cortex. Neural Comput 14:493-525.

Bringuier V, Chavane F, Glaeser L, Frégnac Y (1999) Horizontal propagation of visual activity in the synaptic integration field of area 17 neurons. Science 283:695-699.

Bullier J, Hupe JM, James AC, Girard P (2001) The role of feedback connections in shaping the responses of visual cortical neurons. Prog Brain Res 134:193-204.

Cavanaugh JR, Bair W, Movshon JA (2002a) Nature and interaction of signals from the receptive field center and surround in macaque V1 neurons. J Neurophysiol 88:2530-2546.

Cavanaugh JR, Bair W, Movshon JA (2002b) Selectivity and spatial distribution of signals from the receptive field surround in macaque V1 neurons. J Neurophysiol 88:2547-2556.

Chisum HJ, Mooser F, Fitzpatrick D (2003) Emergent properties of layer $2 / 3$ neurons reflect the collinear arrangement of horizontal connections in tree shrew visual cortex. J Neurosci 23:2947-2960.

DeAngelis GC, Robson JG, Ohzawa I, Freeman RD (1992) Organization of suppression in receptive fields of neurons in cat visual cortex. J Neurophysiol 68:144-163.

DeAngelis GC, Freeman RD, Ohzawa I (1994) Length and width tuning of neurons in the cat's primary visual cortex. J Neurophysiol 71:347-374.

Destexhe A, Rudolph M, Fellous JM, Sejnowski TJ (2001) Fluctuating synaptic conductances recreate in vivo-like activity in neocortical neurons. Neuroscience 107:13-24.

Dragoi V, Sur M (2000) Dynamic properties of recurrent inhibition in primary visual cortex: contrast and orientation dependence of contextual effects. J Neurophysiol 83:1019-1030.

Fitzpatrick D (2000) Seeing beyond the receptive field in primary visual cortex. Curr Opin Neurobiol 10:438-443.

Friedman HS, Zhou H, von der Heydt R (2003) The coding of uniform colour figures in monkey visual cortex. J Physiol (Lond) 548:593-613.

Gilbert CD, Wiesel TN (1990) The influence of contextual stimuli on the orientation selectivity of cells in primary visual cortex of the cat. Vision Res 30:1689-1701.

Gilbert CD, Das A, Ito M, Kapadia M, Westheimer G (1996) Spatial integration and cortical dynamics. Proc Natl Acad Sci USA 93:615-622.

Girard P, Hupé JM, Bullier J (2001) Feedforward and feedback connections between areas V1 and V2 of the monkey have similar rapid conduction velocities. J Neurophysiol 85:1328-1331.

Grinvald A, Lieke EE, Frostig RD, Hildesheim R (1994) Cortical pointspread function and long-range lateral interactions revealed by real-time optical imaging of macaque monkey primary visual cortex. J Neurosci 14:2545-2568.

Hirsch JA, Gilbert CD (1991) Synaptic physiology of horizontal connections in the cat's visual cortex. J Neurosci 11:1800-1809.
Hubel DH, Wiesel TN (1962) Receptive fields, binocular interaction and functional architecture in the cat's visual cortex. J Physiol (Lond) 160:106-154.

Hupé JM, James AC, Payne BR, Lomber SG, Girard P, Bullier J (1998) Cortical feedback improves discrimination between figure and background by V1, V2 and V3 neurons. Nature 394:784-787.

Ichida JM, Schwabe L, Bressloff PC, Angelucci A (2005) Feedback-mediated facilitation and suppression from the receptive field surround of macaque V1 neurons. Soc Neurosci Abstr 31:820.4.

Johnson RR, Burkhalter A (1996) Microcircuitry of forward and feedback connections within rat visual cortex. J Comp Neurol 368:383-398.

Kapadia MK, Ito M, Gilbert CD, Westheimer G (1995) Improvement in visual sensitivity by changes in local context: parallel studies in human observers and in V1 of alert monkeys. Neuron 15:843-856.

Kapadia MK, Westheimer G, Gilbert CD (1999) Dynamics of spatial summation in primary visual cortex of alert monkeys. Proc Natl Acad Sci USA 96:12073-12078.

Kapfer C, Pouille F, Scanziani M (2005) Recurrent inhibition in layer 2/3 of the somatosensory cortex. Soc Neurosci Abstr 31:968.7.

Kennedy H, Bullier J (1985) A double-labeling investigation of the afferent connectivity to cortical area V1 and V2 of the macaque monkey. J Neurosci 5:2815-2830.

Kisvárday ZF, Martin KA, Freund TF, Magloczky Z, Whitteridge D, Somogyi P (1986) Synaptic targets of HRP-filled layer III pyramidal cells in the cat striate cortex. Exp Brain Res 64:541-552.

Lee TS, Mumford D, Romero R, Lamme VA (1998) The role of the primary visual cortex in higher level vision. Vis Res 38:2429-2454.

Levitt JB, Lund JS (1997) Contrast dependence of contextual effects in primate visual cortex. Nature 387:73-76.

Levitt JB, Lund JS (2002) The spatial extent over which neurons in macaque striate cortex pool visual signals. Vis Neurosci 19:439-452.

Li C, Li W (1994) Extensive integration field beyond the classical receptive field of cat's striate cortical neurons: classification and tuning properties. Vision Res 34:2337-2355.

Lund JS, Wu Q, Hadingham PT, Levitt JB (1995) Cells and circuits contributing to functional properties in area V1 of macaque monkey cerebral cortex: bases for neuroanatomically realistic models. J Anat 187:563-581.

Markram H, Wang Y, Tsodyks M (1998) Differential signaling via the same axon of neocortical pyramidal neurons. Proc Natl Acad Sci USA 95:5323-5328.

Martin KA, Whitteridge D (1984) Form, function and intracortical projections of spiny neurons in the striate cortex of the cat. J Physiol (Lond) 353:463-504.

McGuire BA, Gilbert CD, Rivlin PK, Wiesel TN (1991) Targets of horizontal connections in macaque primary visual cortex. J Comp Neurol 305:370-392.

Mignard M, Malpeli JG (1991) Paths of information flow through visual cortex. Science 251:1249-1251.

Movshon JA, Newsome WT (1996) Visual response properties of striate cortical neurons projecting to area MT in macaque monkeys. J Neurosci 16:7733-7741.

Muller JR, Metha AB, Krauskopf J, Lennie P (2003) Local signals from beyond the receptive fields of striate cortical neurons. J Neurophysiol 90:822-831.

Nelson JI, Frost B (1978) Orientation selective inhibition from beyond the classical receptive field. Brain Res 139:359-365.

Nowak LG, Bullier J (1997) The timing of information transfer in the visual system. In: Extrastriate cortex in primates (Rockland KS, Kaas JH, Peters A, eds), pp 205-241. New York: Plenum.

Ozeki H, Sadakane O, Akasaki T, Naito T, Shimegi S, Sato H (2004) Relationship between excitation and inhibition underlying size tuning and contextual response modulation in the cat primary visual cortex. J Neurosci 24:1428-1438.

Polat U, Mizobe K, Pettet MW, Kasamatsu T, Norcia AM (1998) Collinear stimuli regulate visual responses depending on cell's contrast threshold. Nature 391:580-584.

Pouille F, Scanziani M (2004) Routing of spike series by dynamic circuits in the hippocampus. Nature 429:717-723.

Sandell JH, Schiller PH (1982) Effect of cooling area 18 on striate cortex cells in the squirrel monkey. J Neurophysiol 48:38-48.

Sceniak MP, Ringach DL, Hawken MJ, Shapley R (1999) Contrast's effect on spatial summation by macaque V1 neurons. Nat Neurosci 2:733-739. 
Sceniak MP, Hawken MJ, Shapley RM (2001) Visual spatial characterization of macaque V1 neurons. J Neurophysiol 85:1873-1887.

Schwabe L, Obermayer K (2005) Adaptivity of tuning functions in a generic recurrent network model of a cortical hypercolumn. J Neurosci 25:3323-3332.

Sengpiel F, Sen A, Blakemore C (1997) Characteristics of surround inhibition in cat area 17. Exp Brain Res 116:216-228.

Sengpiel F, Baddeley RJ, Freeman TC, Harrad R, Blakemore C (1998) Different mechanisms underlie three inhibitory phenomena in cat area 17. Vision Res 38:2067-2080.

Shao Z, Burkhalter A (1996) Different balance of excitation and inhibition in forward and feedback circuits of rat visual cortex. J Neurosci 16:7353-7365.

Shapley R (2004) A new view of the primary visual cortex. Neural Netw 17:615-623.

Shmuel A, Korman M, Sterkin A, Harel M, Ullman S, Malach R, Grinvald A (2005) Retinotopic axis specificity and selective clustering of feedback projections from V2 to V1 in the owl monkey. J Neurosci 25:2117-2131.

Shriki O, Hansel D, Sompolinsky H (2003) Rate models for conductancebased cortical neuronal networks. Neural Comput 15:1809-1841.

Sillito AM, Cudeiro J, Murphy PC (1993) Orientation sensitive elements in the corticofugal influence on centre-surround interactions in the dorsal lateral geniculate nucleus. Exp Brain Res 93:6-16.

Sincich LC, Blasdel GG (2001) Oriented axon projections in primary visual cortex of the monkey. J Neurosci 21:4416-4426.

Solomon SG, White AJR, Martin PR (2002) Extra-classical receptive field properties of parvocellular, magnocellular, and koniocellular cells in the primate lateral geniculate nucleus. J Neurosci 22:338-349.

Somers DC, Nelson SB, Sur M (1995) An emergent model of orientation selectivity in cat visual cortical simple cells. J Neurosci 15:5448-5465.
Somers DC, Todorov EV, Siapas AG, Toth LJ, Kim DS, Sur M (1998) A local circuit approach to understanding integration of long-range inputs in primary visual cortex. Cereb Cortex 8:204-217.

Stetter M, Bartsch H, Obermayer K (2000) A mean-field model for orientation tuning, contrast saturation, and contextual effects in the primary visual cortex. Biol Cybern 82:291-304.

Stettler DD, Das A, Bennett J, Gilbert CD (2002) Lateral connectivity and contextual interactions in macaque primary visual cortex. Neuron 36:739-750.

Thomson AM (2003) Presynaptic frequency- and pattern-dependent filtering. J Comput Neurosci 15:159-202.

Thomson AM, West DC, Deuchars J (1995) Properties of single axon excitatory postsynaptic potentials elicited in spiny interneurons by action potentials in pyramidal neurons in slices of rat neocortex. Neurosci 69:727-738.

Thomson AM, Bannister AP, Mercer A, Morris OT (2002) Target and temporal pattern selection at neocortical synapses. Philos Trans R Soc Lond B Biol Sci 357:1781-1791.

Van Essen DC, Newsome WT, Maunsell JH (1984) The visual field representation in striate cortex of the macaque monkey: asymmetries, anisotropies, and individual variability. Vision Res 24:429-448.

Walker GA, Ohzawa I, Freeman RD (2000) Suppression outside the classical cortical receptive field. Vis Neurosci 17:369-379.

Webb BS, Dhruv NT, Solomon SG, Taliby C, Lennie P (2005) Early and late mechanisms of surround suppression in striate cortex of macaque. J Neurosci 25:11666-11675.

Weliky M, Kandler K, Fitzpatrick D, Katz LC (1995) Patterns of excitation and inhibition evoked by horizontal connections in visual cortex share a common relationship to orientation columns. Neuron 15:541-552. 